\title{
A comparison of pre-impact gas cushioning and Wagner theory for liquid-solid impacts
}

Cite as: Phys. Fluids 31, 042101 (2019); https://doi.org/10.1063/1.5086510

Submitted: 21 December 2018 . Accepted: 11 March 2019 . Published Online: 02 April 2019

Snizhana Ross (D), and Peter D. Hicks (D)
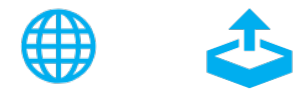

\section{ARTICLES YOU MAY BE INTERESTED IN}

Dynamic behavior of water drops impacting on cylindrical superhydrophobic surfaces

Physics of Fluids 31, 032104 (2019); https://doi.org/10.1063/1.5083070

Numerical study of the shear-thinning effect on the interaction between a normal shock wave and a cylindrical liquid column

Physics of Fluids 31, 043101 (2019); https://doi.org/10.1063/1.5083633

Construction of knotted vortex tubes with the writhe-dependent helicity

Physics of Fluids 31, 047101 (2019); https://doi.org/10.1063/1.5088015

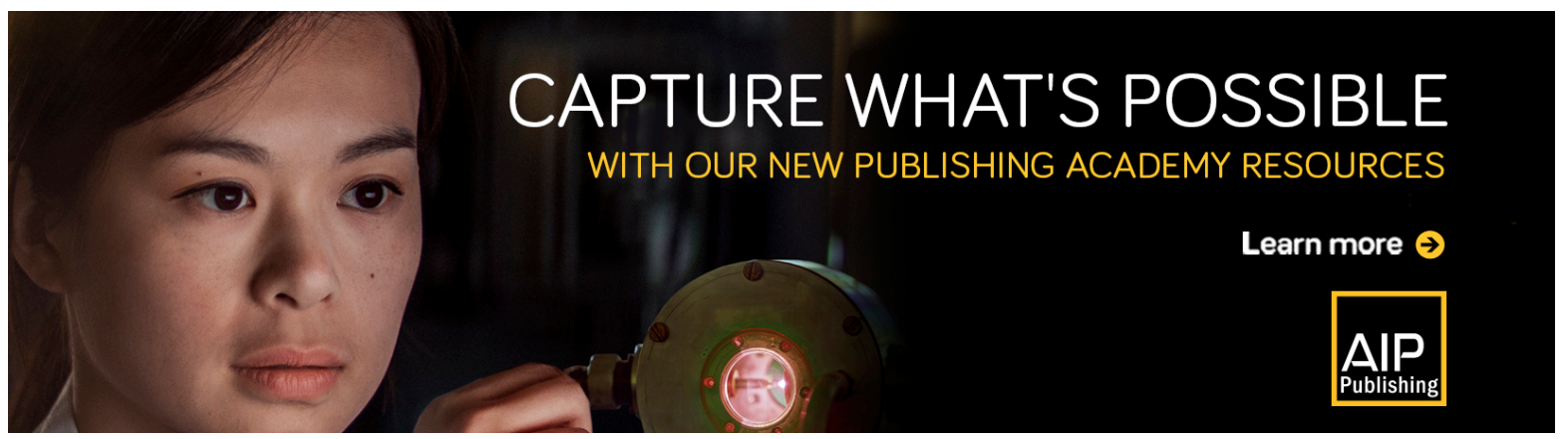




\title{
A comparison of pre-impact gas cushioning and Wagner theory for liquid-solid impacts
}

\author{
Cite as: Phys. Fluids 31, 042101 (2019); doi: 10.1063/1.5086510 \\ Submitted: 21 December 2018 - Accepted: 11 March 2019 • \\ Published Online: 2 April 2019
}

\author{
Snizhana Ross (i) and Peter D. Hicks
}

\section{AFFILIATIONS}

School of Engineering, University of Aberdeen, King's College, Fraser Noble Building, Aberdeen AB24 3 UE, United Kingdom

a)p.hicks@abdn.ac.uk

\begin{abstract}
The normal impact of a symmetric rigid body with an initially quiescent liquid half-space is considered using both Wagner theory and a model of viscous gas pre-impact cushioning. The predictions of these two theories are compared for a range of different body shapes. Both theories assume that the impactor has small deadrise angle. Novel solutions of the Wagner normal impact problem for a symmetric body with a power-law shape are presented, which generalize the well-known results for a parabola and a wedge. For gas cushioned pre-impacts, it is shown that a pocket of gas is entrained even for body shapes with a cusp at the body minimum. A scaling law is developed that relates the dimensions of the trapped gas pocket to the slope of the body. For pre-impact gas cushioning, surface tension is shown to smooth the liquid free-surface and delay the instant of touchdown for a smooth parabolic body, while for a wedge, increasing surface tension initially delays touchdown, before hastening touchdown as the importance of surface tension is increased further. For a flat-bottomed wedge, gas entrainment is again predicted in the gas-cushioning model although the location of initial touchdown, either on the transition between the wedge and the flat bottom or along the side of the wedge, now depends upon the parameters of the body shape.
\end{abstract}

Published under license by AIP Publishing. https://doi.org/10.1063/1.5086510

\section{INTRODUCTION}

Since the pioneering work by Wagner, ${ }^{1}$ which investigated the loads experienced by seaplanes landing upon water, many authors have sought to characterize the pressures and loads generated by impacts between solid bodies and liquids in the context of ship slamming, naval architecture, and aircraft ditching. If gravity, surface tension, and viscous effects are neglected, then the theory of matched asymptotic expansions has facilitated and formalized the development of Wagner theory, which seeks to predict the pressure, the impact load, and the free-surface evolution for small times after the initial impact between liquid and body. ${ }^{2-6}$ More recently this theory has been applied to smaller scale droplet impacts and splashing.

Wagner theory traditionally assumes the role of the surrounding gas is negligible. However, models of pre-impact gas cushioning have also been developed and solved numerically to investigate how a gas pressure buildup prior to impact may cushion the impact and deform the liquid free-surface before the solid body contacts the liquid. Gas cushioning is important in ship slamming, where it has been shown that gas cushioning can reduce the maximum impact pressures for bodies with deadrise angles of less than three degrees. ${ }^{10-12}$ Trapped air can also significantly influence wave impacts on walls, which has important implications for the construction of coastal defences ${ }^{13}$ and determining sloshing behavior during the transport of liquefied natural gas (LNG). ${ }^{14}$ The earliest models of gas cushioning assume inviscid gas behavior. ${ }^{15,16}$ However, more recently viscous effects in the gas have been shown to be significant. ${ }^{17-19}$ Models of pre-impact viscous gas cushioning are usually developed to describe bubble entrapment in droplet impacts and have been extended to incorporate gas compression, ${ }^{20}$ surface tension, ${ }^{18,21}$ and impacts with porous surfaces. ${ }^{22}$ However, they have also been applied and successfully compared to experiments of solid body impacts with a quiescent liquid. ${ }^{23,24}$ For droplet impacts, while a reduction in the surrounding gas pressure suppresses splash jet formation, ${ }^{25}$ it does not eliminate the formation of the trapped gas bubble beneath the droplet, ${ }^{26}$ which has important implications for bubble entrapment in spray coated surfaces. Consequently, understanding how gas influences impact dynamics is important in many practical situations.

Unlike Wagner theory, in which touchdown occurs the instant the solid body reaches the undisturbed liquid free-surface, models of 
gas-cushioned impact indicate that there is a delay in the instant of touchdown due to the free surface being deformed out of the path of the impactor. This implies that there exists a time interval after the initial touchdown in Wagner theory and before the delayed touchdown in models of gas cushioning, where, subject to their respective assumptions, both theories hold. It is in this interval that we shall investigate the similarities and differences between the two theories, as well as highlight cases where gas cushioning is expected to play a significant role in the impact dynamics.

Although Wagner theory historically does not consider the gas phase, attempts have been made to extend Wagner theory by incorporating gas effects into post impact behavior. These attempts have assumed both viscous ${ }^{18}$ and inviscid ${ }^{27,28}$ gas behavior. However, existing models of gas cushioning in post-impact assume that there is no gas pressure buildup in the pre-impact phase, with the liquid free-surface remaining undisturbed until touchdown. Consequently, these extensions of Wagner theory are unable to describe the postimpact evolution of the pocket of trapped gas, which is observed in models of pre-impact gas cushioning. Conversely, numerical solutions of pre-impact cushioning models are only valid up to the point of first contact between liquid and solid and hence cannot currently describe post-impact dynamics.

Section II describes the derivation of a model of viscous gascushioned impact between a solid body and an initially quiescent inviscid liquid, as well as the assumptions under which this model is valid. Section III describes Wagner theory for small times after impact in the absence of gas cushioning. Section IV investigates and compares gas-cushioned impact model behavior with Wagner theory for a range of different body shapes including a quadratic body (Sec. IV A), a wedge (Sec. IV B), a power-law shaped body (Sec. IV C), and a flat-bottomed wedge (Sec. IV D). Conclusions and further discussions are given in Sec. $V$.

\section{PRE-IMPACT GAS CUSHIONING}

The influence of gas cushioning on the impact dynamics of a symmetric body approaching a normal impact with a liquid freesurface (as illustrated in Fig. 1 ) will be investigated. For an incompressible Newtonian fluid, the velocity $\tilde{\boldsymbol{u}}$, with components $(\tilde{u}, \tilde{v})$ in the $(\tilde{x}, \tilde{y})$ direction satisfies the Navier-Stokes equations

$$
\tilde{\nabla} \cdot \tilde{\boldsymbol{u}}=0,
$$

$$
\frac{\partial \tilde{\boldsymbol{u}}}{\partial \tilde{t}}+\tilde{\boldsymbol{u}} \cdot \tilde{\nabla} \tilde{\boldsymbol{u}}=-\frac{1}{\rho} \tilde{\nabla} \tilde{p}+\frac{\mu}{\rho} \tilde{\nabla}^{2} \tilde{\boldsymbol{u}} .
$$

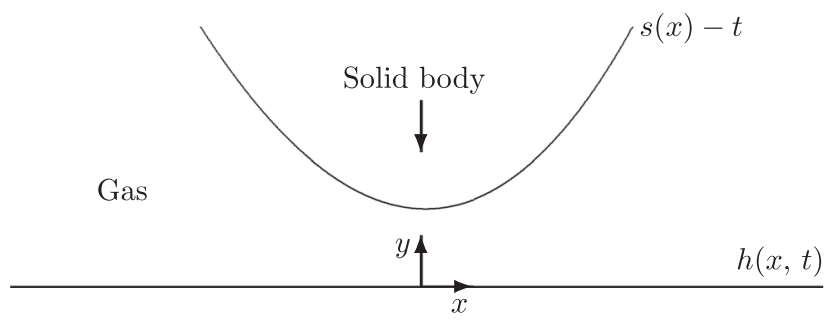

Liquid

FIG. 1: A gas-cushioned liquid-solid impact for the normal impact of a symmetric body.
Here, $\tilde{p}$ is the fluid pressure, $\rho$ is the fluid density, $\mu$ is the fluid viscosity, and $\tilde{t}$ is the time. A solid body with boundary $\tilde{s}(\tilde{x})-V \tilde{t}$ and characteristic length scale $L$ moves normally toward the undisturbed liquid free-surface with uniform velocity $V$. Subsequently, a property of the liquid will be denoted by a subscript $l$, while a subscript $g$ will denote a property of the gas. On the boundary of the solid body $\tilde{y}=\tilde{s}(\tilde{x})-V \tilde{t}$, no-slip and no-penetration boundary conditions imply $\left(\tilde{u}_{g}, \tilde{v}_{g}\right)=(0,-V)$, while on the liquid free-surface $\tilde{y}=\tilde{h}(\tilde{x}, \tilde{t})$, the kinematic boundary condition implies $\tilde{\boldsymbol{u}}_{g}=\tilde{\boldsymbol{u}}_{l}$. The interfacial stresses are additionally balanced across the liquid free-surface. Here, a tilde represents a dimensional variable.

It is assumed that the gas pressure buildup in the gas does not influence the liquid until the vertical separation between the solid body and the liquid free-surface is of size $\varepsilon^{2} L$, where $\varepsilon$ is a small parameter whose value is to be determined. For bodies of small deadrise angle, the horizontal extent of the gas cushioning region is larger and will be assumed to be of size $\varepsilon L$. However, the horizontal extent of the cushioning region is still much smaller than the characteristic length scale of the body $L$, and consequently, gas cushioning only effects a small region about the location with least separation between the solid body and the liquid free-surface. The characteristic time scale for the problem is $\varepsilon^{2} L / V$, which is the time taken for the solid body to transcend the gas cushion in the absence of freesurface deformation. Driven by the descent speed of the body, the characteristic vertical and horizontal velocities in the liquid and the characteristic vertical velocity in the gas are taken to be $V$. However, given the small aspect ratio of the gas cushion, the horizontal gas velocity must be faster (with characteristic velocity $V / \varepsilon$ ) to preserve the mass of gas.

Given the characteristic time scale and velocity in the liquid, a liquid pressure increase from the ambient pressure of magnitude $\rho_{l} V^{2} / \varepsilon$ is required to maintain a leading order contribution from the pressure in the Navier-Stokes equations (1) for the liquid. For pressures smaller than this characteristic scale, there is insufficient pressure buildup at the liquid free-surface to induce liquid motion. Coupled interactions between the gas and liquid are required for gas cushioning, and consequently an equivalent characteristic pressure scale is required in the gas so that the two fluids are coupled through the normal stress balance across the liquid free-surface. With this pressure scale in the gas, the leading-order behavior in the horizontal momentum conservation equation is governed by a balance between the pressure gradient and the vertical viscous term. This balance implies ${ }^{19}$

$$
\varepsilon=\left(\frac{\mu_{g}}{\rho_{l} V L}\right)^{1 / 3} .
$$

For water and air, the impact of a solid body with $L=1 \mathrm{~m}$ and $V=0.1 \mathrm{~m} \mathrm{~s}^{-1}$ results in $\varepsilon=0.0056$, validating the assumption that $\varepsilon$ is a small parameter. The viscous gas can be assumed to be incompressible if the characteristic gas pressure $\rho_{l} V^{2} / \varepsilon$ is smaller than the ambient gas pressure $p_{0}$. This condition is satisfied for impact velocities $^{20}$

$$
V \ll V_{\text {comp }}=\left(\frac{p_{0}^{3} \mu_{g}}{\rho_{l}^{4} R}\right)^{1 / 7},
$$

and consequently, the gas can be assumed to be incompressible for impact velocities in this range. For water and air, $V_{\text {comp }}=0.56 \mathrm{~m} \mathrm{~s}^{-1}$ 
for bodies with characteristic length $L=1 \mathrm{~m}$. The liquid is assumed to be incompressible throughout.

If the Navier-Stokes equations (1) are non-dimensionalized in both the liquid and the gas, using the scales described, then for $\rho_{g} / \rho_{l} \ll \varepsilon$ and $\varepsilon \ll 1$, the leading-order behavior in the gas layer is governed by

$$
\begin{gathered}
\frac{\partial u_{g}}{\partial x}+\frac{\partial v_{g}}{\partial y}=0 \\
-\frac{\partial p_{g}}{\partial x}+\frac{\partial^{2} u_{l}}{\partial y^{2}}=0 \\
-\frac{\partial p_{g}}{\partial y}=0
\end{gathered}
$$

while for a Reynolds number $\operatorname{Re}_{l}=\rho_{l} V L / \mu_{g} \gg 1$ and $\varepsilon \ll 1$, the leading-order behavior in the liquid is governed by the linearized Euler equations

$$
\begin{gathered}
\frac{\partial u_{l}}{\partial x}+\frac{\partial v_{l}}{\partial y}=0 \\
\frac{\partial u_{l}}{\partial t}=-\frac{\partial p_{l}}{\partial x}
\end{gathered}
$$

and

$$
\frac{\partial v_{l}}{\partial t}=-\frac{\partial p_{l}}{\partial y}
$$

Here, variables without a tilde are non-dimensional. For $V=0.1 \mathrm{~m} \mathrm{~s}^{-1}$ and $L=1 \mathrm{~m}, \operatorname{Re}_{l} \approx 100000$, and $\rho_{g} /\left(\rho_{l} \varepsilon\right) \approx 0.15$, which justifies the simplifications described above as the terms neglected are smaller than the terms retained. Assuming that the vertical height of the disturbances to the liquid free-surface are of size $\varepsilon^{2} L$, i.e., a $\mathrm{O}(\varepsilon)$ smaller than the characteristic vertical extent of the liquid, the liquid evolution can be determined on the lower half-space to leading order.

The vertical momentum equation in the gas film (4c) implies $p_{g}=p_{g}(x, t)$, and consequently, upon integrating the mass conservation equation and the horizontal momentum equation from the liquid free-surface $y=h(x, t)$ to the solid body position $y=s(x)-t$, it is found that the rate of change of the free-surface height and the gas pressure is related through the equation

$$
\frac{\partial h}{\partial t}+1=-\frac{1}{12} \frac{\partial}{\partial x}\left[(s(x)-t-h)^{3} \frac{\partial p_{g}}{\partial x}\right]
$$

From the leading order liquid behavior, the acceleration of the liquid free-surface can be related to the liquid pressure at the free surface using either Green's function ${ }^{16}$ or a complex variable approach ${ }^{17}$ in the lower half-space occupied by the liquid through the expression

$$
\frac{\partial^{2} h}{\partial t^{2}}=-\frac{1}{\pi} f_{-\infty}^{\infty} \frac{p_{l, \xi} \mathrm{d} \xi}{x-\xi} .
$$

Here, the kinematic boundary conditions have been used to relate the interfacial fluid velocities to temporal derivatives of the freesurface position. Compared to the earlier analysis of gas-cushioned droplet impacts by Smith, $\mathrm{Li}$, and $\mathrm{Wu},{ }^{17}$ this equation has an additional minus sign, as the positions of the fluids are reversed, with the liquid now occupying the lower half-space and having an outward pointing unit normal in the positive $y$ direction.
The gas and liquid pressures in Eqs. (6) and (7) are coupled across the liquid free-surface by the normal stress balance. At the interface, there is a discontinuity in the normal stresses between the liquid and gas, and this difference equals the dimensional surface tension coefficient $\tilde{\sigma}$, multiplied by the curvature of the interface. With the non-dimensionalization described herein, this implies

$$
p_{l}=p_{g}-\sigma \frac{\partial^{2} h}{\partial x^{2}}
$$

to the leading order, where the reciprocal of the reduced Weber number $\sigma=\varepsilon /$ We. Here, the Weber number We $=\rho_{l} V^{2} L / \tilde{\sigma}$. For $V=0.1 \mathrm{~m} \mathrm{~s}^{-1}$ and $L=1 \mathrm{~m}, \sigma \approx 4 \times 10^{-5}$, indicating that surface tension should be neglected for impacts of this size. However, for slower impact velocities $\left(V=0.02 \mathrm{~m} \mathrm{~s}^{-1}\right)$, involving smaller bodies $(L=0.1 \mathrm{~m}), \sigma \approx 0.04$. It will be shown that surface tension parameters of this size can have a significant influence on the impact dynamics and so the surface tension term is retained to facilitate analysis of smaller scale impacts. For $V=0.02 \mathrm{~m} \mathrm{~s}^{-1}$ and $L=0.1 \mathrm{~m}, \varepsilon \approx 0.02, \rho_{g} /\left(\rho_{l} \varepsilon\right) \approx 0.06$ and $\mathrm{Re}_{l} \approx 2000$, and so the assumptions underpinning the reduction of the Navier-Stokes equations remain valid.

Given a prescribed non-dimensional body position $y=s(x)-t$, Eqs. (6)-(8) form a closed system of equations governing twodimensional pre-impact cushioning of a liquid-solid impact. With surface tension included, the boundary integral equation (7) and normal stress balance (8) are efficiently solved by taking Fourier transforms of both the pressure and free-surface. ${ }^{22}$ This is coupled to an implicit finite difference solver for the Reynolds squeeze film Eq. (6).

To facilitate a direct comparison with Wagner theory, it is expedient to define both the liquid velocity potential $\phi_{l}$ and the liquid displacement potential $\Phi_{l}$. For $\varepsilon \ll 1$, the liquid velocity potential and the liquid pressure are connected through the linearized Bernoulli equation

$$
\frac{\partial \phi_{l}}{\partial t}=-p_{l}
$$

while following Korobkin, ${ }^{29}$ the liquid displacement potential is defined to be

$$
\frac{\partial^{2} \Phi_{l}}{\partial t^{2}}=p_{l}
$$

Upon integrating these expressions,

$$
\phi_{l}(x, y, t)=-\int_{-\infty}^{t} p_{l}(x, y, \tau) \mathrm{d} \tau,
$$

and

$$
\Phi_{l}(x, y, t)=-\int_{-\infty}^{t} \phi_{l}(x, y, \tau) \mathrm{d} \tau .
$$

These integrals differ from those proposed by Korobkin, ${ }^{30}$ as the pre-impact gas pressure buildup induces liquid motion from large negative times, rather than just from $t=0$, which is the case for Wagner theory. However, in the absence of gas cushioning, $p_{l}(x, 0, t)=0$ for $-\infty<t<0$, so the liquid remains at rest until $t=0$ and the usual integral relations are recovered. 


\section{WAGNER THEORY}

Wagner theory for the normal impact of a symmetric solid body with an incompressible quiescent liquid is now reviewed. Wagner theory is used to investigate post-impact behavior, as shown in Fig. 2(a). The liquid non-dimensionalization used in Sec. II is again employed, while the impact is assumed to occur in a vacuum, so the corresponding non-dimensionalization of the gas phase is not required. In the absence of gas cushioning, the solid body described in Sec. II impacts a quiescent liquid at $x=y=0$ and time $t=0$ as there is no gas pressure buildup and $p_{l}(x, 0, t)=0$ for $t<0$. Assuming a small deadrise angle for the body, the horizontal extent of the wetted region between body and liquid is much smaller than the penetration depth, ${ }^{4}$ and consequently, the disparate vertical and horizontal length scales used to non-dimensionalize the body position in gascushioned impacts are again employed, guaranteeing a consistent body shape in both cases.

The liquid free-surface $h(x, t)$ is disturbed by the penetration of the body, leading to turnover points at $x= \pm c(t)$, where the free-surface position becomes multivalued as a result of splash jets ejecting liquid from the path of the body. ${ }^{27}$ The free-surface height below the turnover point tends to zero in the far-field, a long way from the impactor. An outer problem can now be formulated for the liquid velocity potential, connecting the turnover point position, the free surface $h(x, t)$ below the turnover point, and the body position. Like the liquid problem for gas cushioning, the Wagner outer problem for small times and penetration depths can be solved in the lower

(a)

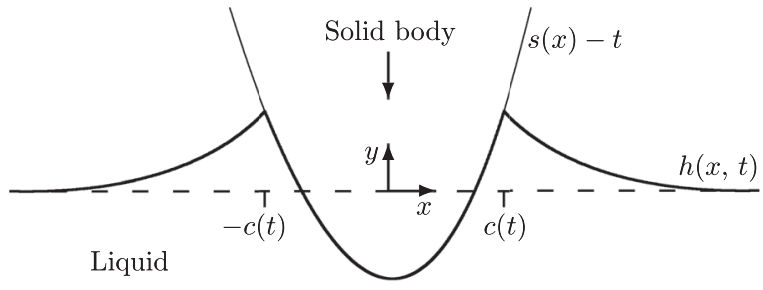

(b)

$$
\begin{gathered}
\phi_{l}=0, \frac{\partial \phi_{l}}{\partial y}=\frac{\partial h}{\partial t} \underbrace{\frac{\partial \phi_{l}}{\partial y}=-1}_{c(t)} \underset{l}{c(t)} \phi_{l}=0, \frac{\partial \phi_{l}}{\partial y}=\frac{\partial h}{\partial t} \\
\frac{\partial^{2} \phi_{l}}{\partial x^{2}}+\frac{\partial^{2} \phi_{l}}{\partial y^{2}}=0
\end{gathered}
$$

(c) $\frac{\Phi_{l}=0, \frac{\partial \Phi_{l}}{\partial y}=-h \quad \frac{\partial \Phi_{l}}{\partial y}=t-s(x) \quad \Phi_{l}=0, \frac{\partial \Phi_{l}}{\partial y}=-h}{c(t)}$

$$
\frac{\partial^{2} \Phi_{l}}{\partial x^{2}}+\frac{\partial^{2} \Phi_{l}}{\partial y^{2}}=0
$$

FIG. 2. (a) A two-dimensional liquid-solid impact and the corresponding mixed boundary value problem for (b) the liquid velocity potential and (c) the liquid displacement potential. half-space. Local inner analyses are required at the turnover jet root region and in the jet itself to resolve the local liquid behavior close to the turnover point and in the liquid jet. ${ }^{4,5}$ However, on the scale of the outer problem, the liquid free-surface height matches the vertical position of the body at the contact line $x= \pm c(t)$, which gives rise to the Wagner condition

$$
s( \pm c(t))-t=h( \pm c(t), t) .
$$

For the outer problem, the liquid velocity potential satisfies the mixed boundary value problem

$$
\begin{array}{rlrl}
\frac{\partial^{2} \phi_{l}}{\partial x^{2}}+\frac{\partial^{2} \phi_{l}}{\partial y^{2}} & =0, & & y<0, \\
\phi_{l} & =0, & & y=0,|x|>c(t), \\
\frac{\partial \phi_{l}}{\partial y} & =-1, & & y=0,|x|<c(t), \\
\phi_{l} & \rightarrow 0, & \sqrt{x^{2}+y^{2}} \rightarrow \infty,
\end{array}
$$

which is summarized in Fig. 2(b). On the liquid free-surface, the kinematic boundary condition implies

$$
\frac{\partial \phi_{l}}{\partial y}=\frac{\partial h}{\partial t}, \quad \text { for } \quad y=0,|x|>c(t)
$$

The liquid velocity potential $\phi_{l}$ and the stream function $\psi_{l}$ satisfy the Cauchy-Riemann equations. If we define the complex-valued holomorphic function

$$
w_{l}(z, t)=\phi_{l}(x, y, t)+\mathrm{i} \psi_{l}(x, y, t),
$$

where $z=x+\mathrm{i} y$, and then upon substitution in Cauchy's integral formula

$$
\phi_{l, x}(x, y, t)-\mathrm{i} \phi_{l, y}(x, y, t)=\frac{1}{2 \pi \mathrm{i}} \oint_{\gamma} \frac{\phi_{l, \xi}(\xi, \eta, t)-\mathrm{i} \phi_{l, \eta}(\xi, \eta, t)}{\zeta-z} \mathrm{~d} \zeta .
$$

Here, the Cauchy-Riemann equations have been used to eliminate $\psi$, while $\zeta=\xi+\mathrm{i} \eta$. Taking an anticlockwise contour $\gamma$ along $y=0$, followed by a return arc through deep water and noting that $\partial h / \partial t$ is unbounded at $\pm c(t)$, this integral can be inverted to find ${ }^{3}$

$$
\begin{aligned}
\phi_{l, x}(x, y, t)-\mathrm{i} \phi_{l, y}(x, y, t) \\
\quad=\frac{1}{2 \pi \mathrm{i} \Lambda(z)} \oint_{\gamma} \frac{\Lambda(\zeta)\left[\phi_{l, \xi}(\xi, \eta, t)-\mathrm{i} \phi_{l, \eta}(\xi, \eta, t)\right] \mathrm{d} \zeta}{\zeta-z},
\end{aligned}
$$

where, for $z=x-\mathrm{i} 0$, the characteristic function

$$
\Lambda(x)= \begin{cases}-\sqrt{x^{2}-c(t)^{2}} & \text { for } x<-c(t), \\ -\mathrm{i} \sqrt{c(t)^{2}-x^{2}} & \text { for }|x|<c(t), \\ \sqrt{x^{2}-c(t)^{2}} & \text { for } x>c(t) .\end{cases}
$$

In full generality, Eq. (16) includes an additional term equivalent to a constant multiplying the homogeneous solution of this problem. However, if the integral of kinetic energy is to remain bounded, and then this constant is necessarily zero.

For $\zeta=\xi-\mathrm{i} 0$ and $z=x-\mathrm{i} 0$, the imaginary part of Eq. (16) gives

$$
\frac{\partial h}{\partial t}(x, t)=-1+\frac{|x|}{\sqrt{x^{2}-c(t)^{2}}}, \text { for }|x|>c(t)
$$


which matches Eq. 2.41 by Oliver. ${ }^{5}$ Alternatively, for $\zeta=\xi-\mathrm{i} 0$ and $z=x-\mathrm{i} 0$ with $|x|<c(t)$, an expression for $\partial \phi_{l} / \partial x(x, 0, t)$ is recovered from the real part of Eq. (16). Integrating this expression with respect to $x$, before differentiating with respect to $t$, gives the liquid pressure over the wetted surface

$$
p_{l}(x, 0, t)=\frac{c(t) \dot{c}(t)}{\sqrt{c(t)^{2}-x^{2}}}, \quad \text { for } \quad|x|<c(t) .
$$

Here, the dependence of the vertical velocity of the free surface (18) and the liquid pressure (19) on the body shape $s(x)$ is encapsulated in $c(t)$.

A similar analysis can be conducted for the liquid displacement potential $\Phi_{l}$ and its complex conjugate $\Psi_{l}$. The corresponding mixed boundary value problem for the liquid displacement potential satisfies $^{29}$

$$
\begin{array}{rlrl}
\frac{\partial^{2} \Phi_{l}}{\partial x^{2}}+\frac{\partial^{2} \Phi_{l}}{\partial y^{2}} & =0, & & y<0, \\
\Phi_{l} & =0, & & y=0,|x|>c(t), \\
\frac{\partial \Phi_{l}}{\partial y} & =t-s(x), & & y=0,|x|<c(t), \\
\Phi_{l} & \rightarrow 0, & \sqrt{x^{2}+y^{2}} \rightarrow \infty .
\end{array}
$$

This mixed boundary value problem is summarized in Fig. 2(c). Integrating the kinematic boundary condition (13) with respect to time implies

$$
\frac{\partial \Phi_{l}}{\partial y}=-h, \quad \text { on } \quad y=0,|x|>c(t)
$$

If the complex-valued holomorphic function,

$$
W_{l}(z, t)=\Phi_{l}(x, y, t)+\mathrm{i} \Psi_{l}(x, y, t)
$$

is substituted into Cauchy's integral formula, then

$$
\Phi_{l, x}(x, y, t)-\mathrm{i} \Phi_{l, y}(x, y, t)=\frac{1}{2 \pi \mathrm{i}} \oint_{\gamma} \frac{\Phi_{l, \xi}(\xi, \eta, t)-\mathrm{i} \Phi_{l, \eta}(\xi, \eta, t)}{\zeta-z} \mathrm{~d} \zeta .
$$

Expecting $\Phi_{l, x}$ to remain bounded at $x= \pm c(t)$ and using the characteristic function (17), this integral can be inverted to give ${ }^{31}$

$$
\begin{aligned}
\Phi_{l, x}(x, y, t)-\mathrm{i} \Phi_{l, y}(x, y, t) \\
=\frac{\Lambda(z)}{2 \pi \mathrm{i}} \oint_{\gamma} \frac{\left[\Phi_{l, \xi}(\xi, \eta, t)-\mathrm{i} \Phi_{l, \eta}(\xi, \eta, t)\right] \mathrm{d} \zeta}{\Lambda(\zeta)(\zeta-z)},
\end{aligned}
$$

where the consistency condition,

$$
\oint_{\gamma} \frac{\left[\Phi_{l, \xi}(\xi, \eta, t)-\mathrm{i} \Phi_{l, \eta}(\xi, \eta, t)\right] \mathrm{d} \zeta}{\Lambda(\zeta)}=0
$$

must additionally be satisfied.

For $\zeta=\xi-\mathrm{i} 0$ and $z=x-\mathrm{i} 0$ with $|x|>c(t)$, the imaginary part of Eq. (24a) and the real part of Eq. (24b) give

$$
h(x, t)=\frac{\sqrt{x^{2}-c(t)^{2}}}{\pi} \int_{-c(t)}^{c(t)} \frac{t-s(\xi) \mathrm{d} \xi}{\sqrt{c(t)^{2}-\xi^{2}}(\xi-x)}
$$

and

$$
\int_{-c(t)}^{c(t)} \frac{t-s(\xi) \mathrm{d} \xi}{\sqrt{c(t)^{2}-\xi^{2}}}=0
$$

upon the application of the boundary conditions (20c) and (21). A scaled horizontal position $X=x / c(t)$ can be defined, and hence the free-surface position can be expressed as

$$
h(c(t) X, t)=\frac{2 X \sqrt{X^{2}-1}}{\pi} \int_{0}^{1} \frac{s(c(t) \Xi) \mathrm{d} \Xi}{\sqrt{1-\Xi^{2}}\left(\Xi^{2}-X^{2}\right)}-t,
$$

for $X>1$, while the consistency condition implies

$$
\int_{0}^{1} \frac{s(c(t) \Xi) d \Xi}{\sqrt{1-\Xi^{2}}}=\frac{\pi t}{2} .
$$

This scaled horizontal coordinate facilitates the convenient evaluation of these integrals. For a given body shape $s(x)$, the consistency condition can be integrated to determine the contact line position $c(t)$, while the corresponding free-surface height for $|x|>c(t)$ is obtained by substituting for the body shape and integrating either Eq. (25a) or (26a).

\section{RESULTS AND DISCUSSION}

\section{A. Quadratic-shaped body}

The impact of a quadratic parabola-shaped body $s(x)=\alpha x^{2}$ for $\alpha>0$ is investigated first. Given the disparate horizontal and vertical scaling in the gas layer, this body shape is the local approximation of a circular impactor for $\alpha=\frac{1}{2}$ and of an elliptical impactor with semi axes normal $(L)$ and parallel $\left(L_{x}\right)$ to the undisturbed free surface for of $\alpha=L /\left(2 L_{x}\right)$. Consequently, an elliptical impactor is oblate with respect to body motion for $\alpha<\frac{1}{2}$ and is prolate for $\alpha>\frac{1}{2}$. Viscous gas cushioning of liquid-solid impacts with smooth bodies has previously shown good agreement with experiments. ${ }^{23,24}$ However, until now, a comparison of the viscous gas cushioning model and Wagner theory has not been undertaken. Inviscid gas cushioning in impacts of quadratic-shaped bodies has also been investigated.

For a quadratic shaped body $s(x)=x^{2}$, the differences in impact dynamics between pre-impact gas cushioning and Wagner theory are illustrated in Fig. 3. The body and liquid free-surface positions (top left) are shown alongside the corresponding free-surface velocities (top right), liquid pressures at the interface (bottom left), and liquid velocity potentials at the interface (bottom right).

In Wagner theory, touchdown occurs at $t=0$, with the liquid remaining at rest until this time. Wagner theory solutions can be readily obtained for $t>5$ although the final profile is shown at this instant to facilitate direct comparison with pre-impact gas cushioning. For Wagner theory, Eq. (25b) gives rise to the well-known contact line position

$$
c(t)=\sqrt{\frac{2 t}{\alpha}},
$$

while for $|x|>c(t)$, the free-surface height,

$$
h(x, t)=\alpha x^{2}-t-\alpha|x| \sqrt{x^{2}-c(t)^{2}},
$$

is obtained from Eq. (25a). As the body penetrates the liquid, the displaced liquid accumulates around the sides of the body to satisfy 

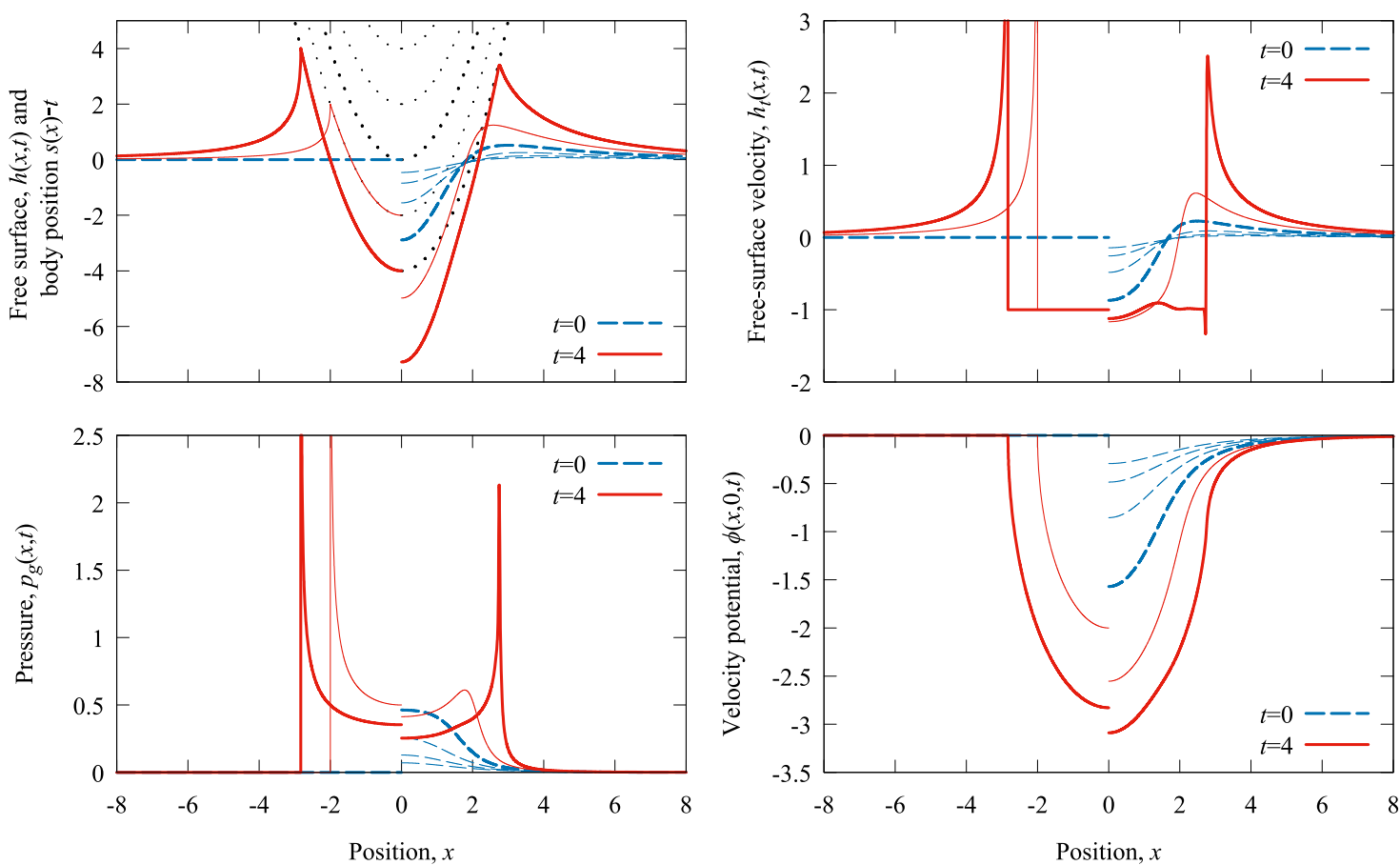

FIG. 3. Free-surface and body position (top left), free-surface velocity (top right), pressure at the free surface (bottom left), and liquid velocity potential at the free surface (bottom right), for the impact of a parabola $s(x)=x^{2}$ (black dotted line). The Wagner solution is shown for $x<0$, while the viscous gas cushioning model is shown for $x>0$. Profiles are shown at every second non-dimensional integer time step. In the absence of gas cushioning, touchdown occurs at $t=0$ (thick dashed blue line), while profiles for $t<0$ are shown as thin dashed blue lines. Profiles for $t>0$ are shown as thin solid red lines, except the final profile (thick solid red line), which is immediately prior to touchdown in a gas-cushioned impact.

the Wagner condition (11). The wetted surface occupies $|x| \leq c(t)$. The leading-order liquid pressure predicted by Wagner theory is unbounded at $x= \pm c(t)$ [see Eq. (19)], and to regularize this pressure, asymptotic analysis in the jet root region surrounding the free-surface turnover point must be conducted. ${ }^{4,5}$ Our interest is in the outer Wagner problem on length scales comparable to preimpact gas cushioning, so the local analysis necessary to regularize the pressure at the contact line is not described.

In gas-cushioned impacts, there is a buildup of pressure in the gas separating the impactor from the liquid free-surface starting from large negative times. This deforms the liquid free-surface, deflecting it out of the path of the oncoming impactor. The liquid moved out of the path of the impactor accumulates some horizontal distance away from the point of initial minimum separation between the solid body and the liquid free-surface, and it is at these points that touchdown initially occurs. After the initial pressure buildup beneath the impactor, the pressure profiles also bifurcate with subsequent pressure maxima occurring where the separation between the body and the liquid free-surface is least. The pressure profiles generated closely match those associated with viscous gas-cushioned droplet impacts as the models of droplet cushioning by viscous gas of Smith, $\mathrm{Li}$, and $\mathrm{Wu},{ }^{17}$ and Purvis and $\mathrm{Smith}^{18}$ are recovered by defining the vertical separation between the solid body and the liquid free-surface to be $f(x, t)=s(x)-t-h(x, t)$ in Eqs. (6) and (7).

Beneath the impactor, a region of trapped gas is formed by the deformation of the liquid free-surface so that the leading-edge of the body is surrounded by gas at touchdown. At the points of initial touchdown, cusps form on the free surface. ${ }^{32}$ The pocket of trapped gas subsequently evolves to form a bubble. ${ }^{23,33}$ Following the scaling argument presented in Sec. II, the horizontal touchdown position $r_{p}$ and maximum vertical height $h_{p}$ of the initial pocket of trapped gas are of the order of the characteristic horizontal and vertical length scales, ${ }^{23,24}$ and consequently,

$$
r_{p}=\varepsilon L r_{p}^{\star}=\left(\frac{\mu_{g} L^{2}}{\rho_{l} V}\right)^{1 / 3} r_{p}^{\star}
$$

and

$$
h_{p}=\varepsilon^{2} L h_{p}^{\star}=\left(\frac{\mu_{g}^{2} L}{\rho_{l}^{2} V^{2}}\right)^{1 / 3} h_{p}^{\star} .
$$

For $\alpha=\frac{1}{2}$, the numerically calculated pre-factors are $r_{p}^{\star} \approx 4.38$ and $h_{p}^{\star} \approx 4.10$, while for $\alpha=1, r_{p}^{\star} \approx 2.78$ and $h_{p}^{\star} \approx 3.29$. For an axisymmetric body $s(r)=\frac{1}{2} r^{2}$, with radial distance $r$, the same scaling laws hold with $r_{p}^{\star} \approx 6$. For an axisymmetric body, the initial radius of the trapped gas pocket has been experimentally measured and excellent agreement with this scaling law has been obtained. ${ }^{23}$ The same scaling laws hold for incompressible, viscous gas-cushioned droplet impacts, where again excellent experimental confirmation of this result has been found. ${ }^{34}$

The similarities and differences between the profiles predicted by Wagner theory and pre-impact gas cushioning are now described. 
To assist this comparison, the horizontal location $c_{\text {gas }}(t)$, of the point of minimum separation between the body and the liquid free-surface in gas-cushioned impacts, is defined to be

$$
c_{\text {gas }}(t) \equiv \min _{x \geq 0}[s(x)-t-h(x, t)] .
$$

Despite the absence of gas cushioning in Wagner theory, there are many similarities between the two different types of the liquid freesurface profile. Both theories conserve the liquid mass moved out of the path of the impactor by predicting a liquid buildup at the sides of the penetrating body. In both Wagner theory [for $|x|>c(t)]$ and in pre-impact cushioning [for $|x|>c_{\text {gas }}(t)$ ], the free-surface decays to its undisturbed value for $|x| \rightarrow \infty$. This decay is faster for Wagner theory [where $\partial h / \partial x$ is unbounded as $x$ approaches $c(t)$ ] than the corresponding decay with pre-impact cushioning (where $\partial h / \partial x$ is bounded throughout). For pre-impact cushioning, a pocket of gas is entrained about the tip of the body [for $|x|<c_{\text {gas }}(t)$ ]. This is not present in Wagner theory as the liquid boundary follows the body shape on the wetted region $|x|<c(t)$, and consequently, at an equivalent time, the disturbance of the liquid surface is greater with pre-impact cushioning.

The pressure predicted by Wagner theory becomes unbounded as $|x| \nearrow c(t)$ and further local asymptotic analysis is required to regularize the pressure in these regions. ${ }^{5}$ However, outside these regions, the pressure profiles predicted by Wagner theory and in pre-impact cushioning can be directly compared. The horizontal location of the maximum pressures is very similar in both models. Also, the pressure obtained at $x=0$ for $t>0$ is of a similar magnitude.

As $|x| \searrow c(t)$, the free-surface velocity predicted by Wagner theory becomes unbounded, like the Wagner pressure. Again, a local asymptotic analysis is required to regularize the free-surface velocity at these points. However, outside a small region surrounding these points, the velocity profiles predicted by Wagner theory can be compared with the corresponding velocity profiles predicted in gas cushioned impacts. For both $x>c(t)$ and $x>c_{\text {gas }}(t)$, the free surface has a positive upwards velocity, which enables the accumulation of liquid around the sides of the impactor. These positive velocities again both decay as $|x| \rightarrow \infty$, enabling the free surface to return to its undisturbed position in the far field. In Wagner theory, the boundary conditions on the mixed boundary value problem for the liquid velocity potential $(12 \mathrm{c})$ equates the downwards velocity of the liquid boundary to the body descent speed for $|x|<c(t)$. As touchdown approaches, the corresponding free-surface velocity with gas cushioning tends toward the same body descent speed for $x<c_{\text {gas }}(t)$, despite the fact that there is now a gas cushion separating liquid from the impactor. The free-surface velocity in the gas entrainment problem matches that predicted by Wagner theory as gas is unable to escape from the region in front of the impactor after the pressure has bifurcated. This is because the pressure gradient is zero at the pressure maxima, and hence there is no gas flux at these points. As the entrained gas is unable to drain out of the path of the impactor, it must be carried along in front of the impactor and naturally, both the gas and the liquid free-surfaces must therefore obtain a vertical velocity comparable to that of the impactor.

Gas-cushioned impacts again exhibit non-zero liquid velocity potentials for $t<0$ due to the gas pressure buildup, which is not present in Wagner theory. However, at the time of touchdown in the gas-cushioned model, the liquid velocity potential on the free surface is very similar in both cases. With gas cushioning, the transition of the liquid velocity potential at $x=c_{\text {gas }}(t)$ is much more gradual, than in Wagner theory, as a result of the smoother pressure. With gas cushioning, the profiles of the liquid velocity potential just before touchdown do not exhibit the same sharp peaks associated with the pressure and the free-surface position, and consequently, calculations based on the liquid velocity potential may be better suited for extension into the post-impact cushioning phase.

In Fig. 3 , it is noticeable that the horizontal location of $c(t)$ and $c_{\text {gas }}(t)$ are similar at touchdown. This similarity between gascushioned behavior and Wagner theory [black dotted line, Eq. (27a)] is illustrated graphically in Fig. 4 for $\alpha=1$. In gas-cushioned impacts $c_{\text {gas }}(t)$ deviates from $x=0$ before $t=0$ due to the bifurcation of the free-surface profile and pressure. For $\sigma=0$ (thick solid blue line), the position $c_{\text {gas }}(t)>c(t)$, until $t \approx 1.7$, when the horizontal motion of $c(t)$ overtakes $c_{\text {gas }}(t)$. Subsequently, the position of $c_{\text {gas }}(t)$ is slightly behind, but closely follows $c(t)$ until touchdown. As surface tension increases $c_{\text {gas }}(t)$ does not deviate from $x=0$ until times close to $t=0$. This is because the capillary pressure dominates over the liquid inertial pressure for large $\sigma$, with the free surface not deforming before touchdown in this limit. The transition to this curvature preserving behavior is observed even for comparatively small $\sigma$. Once $c_{\text {gas }}(t)$ deviates from $x=0$, surface tension acts to prevent the formation of steep gradients on the free surface, and so for a given time, $c_{\text {gas }}(t)$ becomes smaller as $\sigma$ increases. The closest agreement at touchdown is obtained in the absence of surface tension. If the relative difference in the horizontal position of $c(t)$ and $c_{\text {gas }}(t)$ is defined to be

$$
E=100 \% \frac{\left|c(t)-c_{\mathrm{gas}}(t)\right|}{c(t)},
$$

then, in the absence of surface tension, $E=2.88 \%$ at touchdown. The evolution of $c_{\text {gas }}(t)$ is plotted until touchdown, which indicates that surface tension delays the instant of touchdown in gascushioned impacts. No touchdown occurs before $t=24$ when $\sigma=0.04$. With surface tension, greater deviation between $c_{\text {gas }}(t)$ and

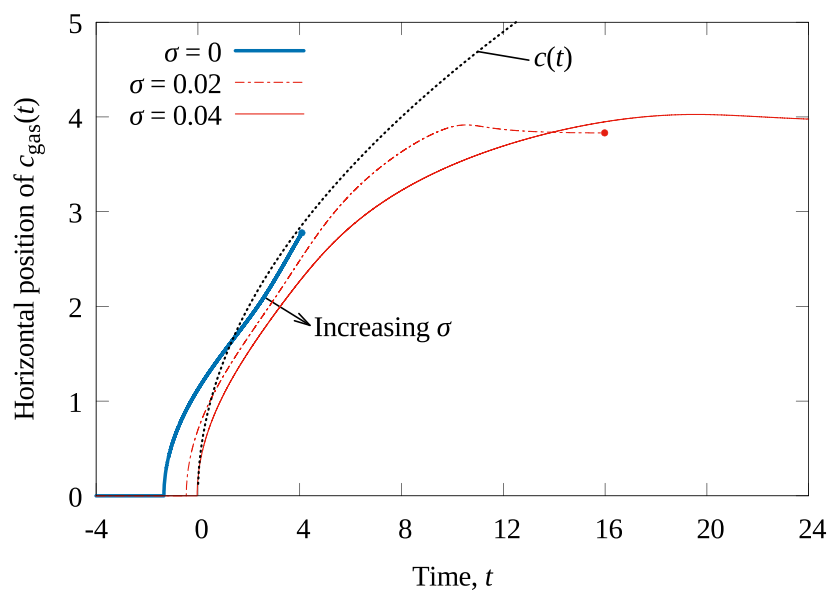

FIG. 4. A comparison of $c_{\text {gas }}(t)$ against $c(t)$ (black dotted line) for a parabola with $\alpha=1$. The position of $c_{\text {gas }}(t)$ is shown for $\sigma=0$ (thick solid blue line), $\sigma=0.02$ (dashed-dotted red line), and $\sigma=0.04$ (thin solid red line). In a gas-cushioned impact, the evolution is shown until the instant of touchdown. 
$c(t)$ is observed at later times, with $c_{\text {gas }}(t)$ obtaining a maximum value at $t=10.54$ for $\sigma=0.02$ and at $t=19.55$ for $\sigma=0.04$, before $c_{\text {gas }}(t)$ begins to move back toward $x=0$.

The influence of surface tension on the free-surface and pressure profiles in gas-cushioned impacts is further illustrated in Fig. 5 for $\alpha=1$ and $\sigma=0,0.02$, and 0.04. Gas-cushioned droplet impacts with surface tension were previously investigated by Purvis and Smith. ${ }^{18}$ As with this earlier study, we find surface tension acts to smooth both the free-surface and pressure profiles, while further delaying the instant of touchdown. This smoothing is particularly noticeable where the curvature of the free surface is greatest. With surface tension, the delay between the instant touchdowns would have occurred in the absence of gas touchdown and the actual touchdown time increases with $\sigma$. There is a corresponding increase in the horizontal extent of the gas pocket, while just prior to touchdown, capillary waves on the free surface are prominent for $\sigma=0.04$.

\section{B. Wedge-shaped body}

Wagner theory and viscous gas-cushioned impacts are now investigated for the wedge $s(x)=\alpha|x|$, with $\alpha>0$. Gas entrapment in impact experiments for rigid wedge-shaped bodies has been observed for deadrise angles of less than $3^{\circ} .{ }^{10,35}$ These very small deadrise angles are consistent with the disparate horizontal and vertical length scales employed herein. However, the presence of the gas is usually inferred from pressure traces, rather than being observed directly. Recent advances in high-speed photography, which have enabled direct measurement of the contact line evolution associated with the gas pocket in the impact of a rounded body, ${ }^{23,33}$ have not been applied to a wedge-shaped body, and consequently, experimental validation of the predicted free-surface profiles in gascushioned wedge impacts is not possible. Impact experiments with wedge-shaped bodies are most often conducted with deadrise angles larger than $3^{\circ}$, and gas entrapment is not observed ${ }^{36-38}$ in this regime. Wilson ${ }^{16}$ models and calculates the free-surface deformation and the horizontal gas profile for a wedge impact cushioned by an inviscid gas. However, results of the viscous gas cushioning model used herein and a direct comparison with Wagner theory have not previously been presented.

Figure 6 shows the evolution of the body and free-surface profile (top left), the free-surface velocity (top right), the pressure at the interface (bottom left), and the liquid velocity potential at the interface (bottom right) for $\alpha=1$. Both Wagner theory (shown for $x<0$ ) and the viscous gas cushioning model (shown for $x>0$ ) assume that the wedge has small deadrise angle. Despite the pointed tip of the wedge, the gas-cushioned impact model predicts that a pocket of gas is entrapped, resulting in a delay to touchdown. The mechanism for this matches that observed with the quadraticshaped body.

For Wagner theory, the wedge initially impacts the liquid at $t=0$, with the liquid at rest before this time. For $t>0$, Wagner theory (a)
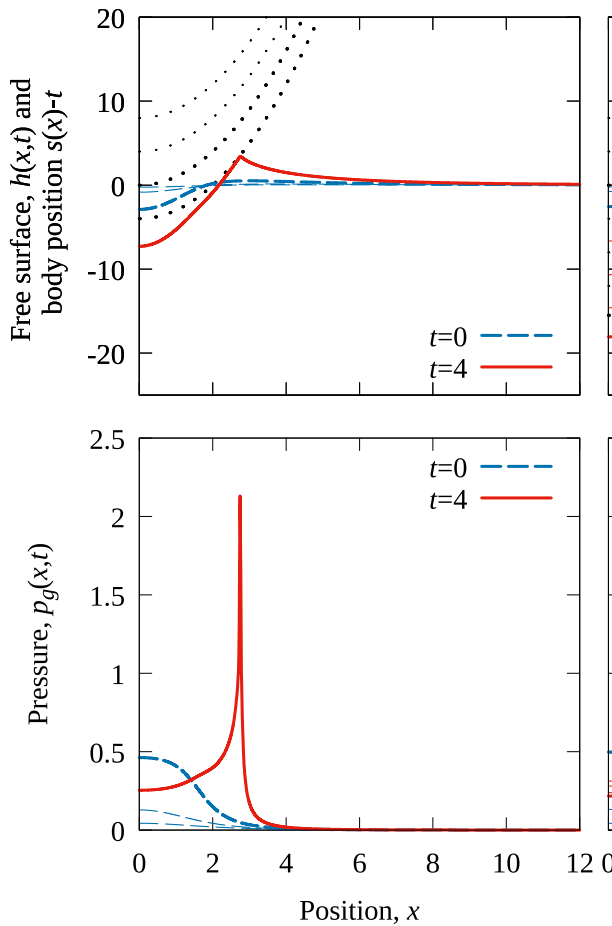

(b)
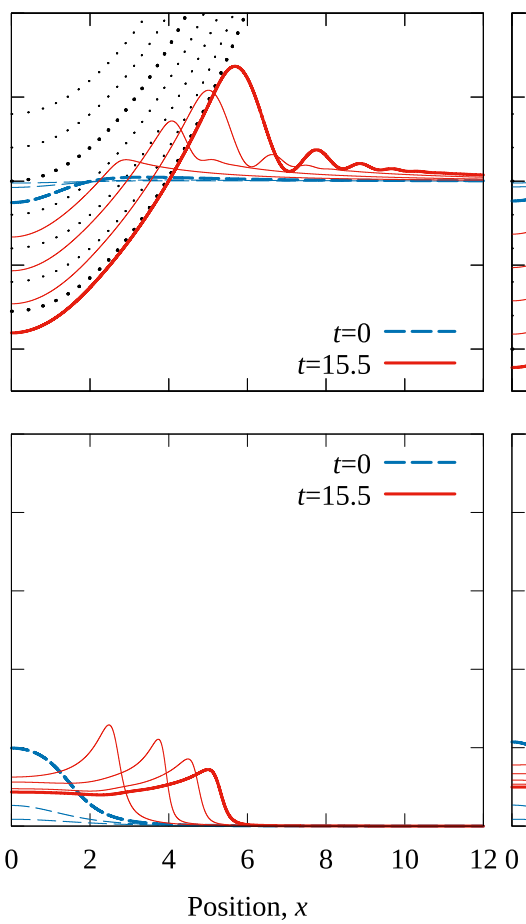

(c)
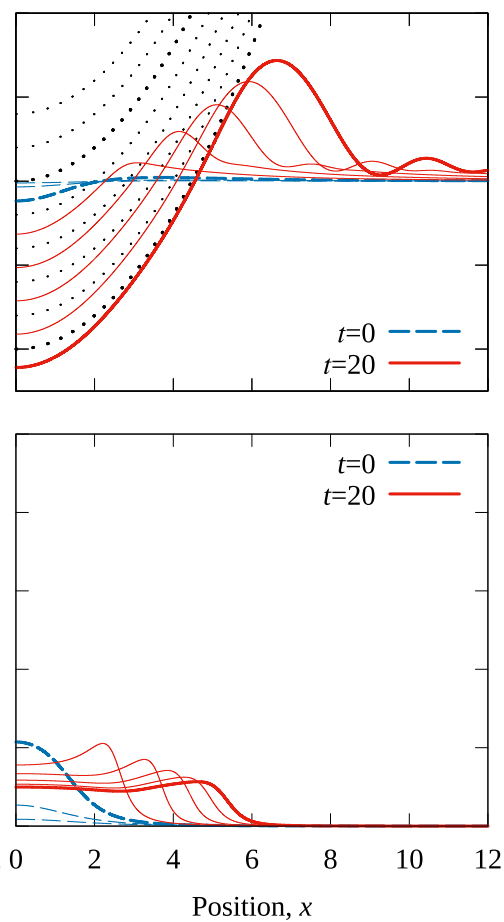

FIG. 5. Gas-cushioned impacts of a quadratic-shaped body $s(x)=x^{2}$ (black dotted line) with surface tension parameters (a) $\sigma=0$, (b) $\sigma=0.02$, and (c) $\sigma=0.04$. In the absence of gas cushioning, touchdown would occur at $t=0$ (thick dashed blue line). Profiles are shown at every fourth non-dimensional integer time step except in (a) and (b), where the final profile (thick solid red line) is shown immediately before touchdown. $\ln (\mathrm{c})$, touchdown does not occur before $t=24$. 

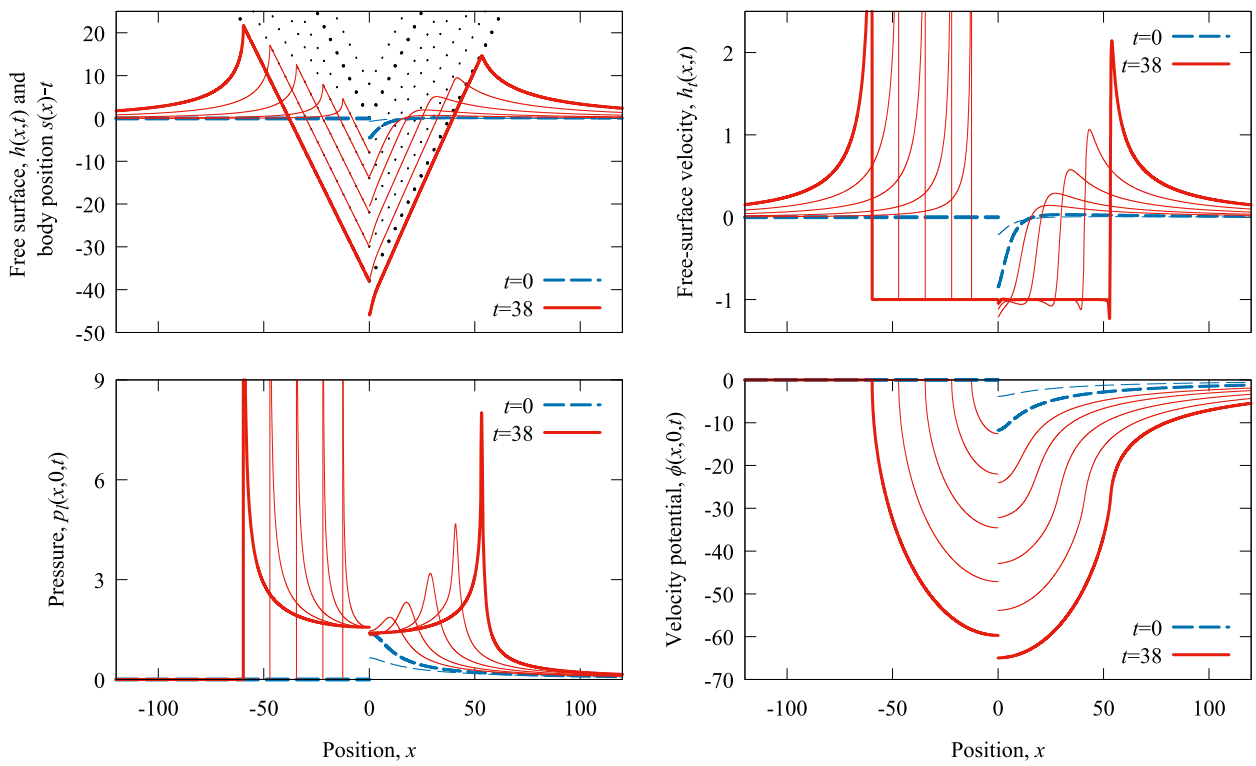

predicts a contact line position

$$
c(t)=\frac{\pi t}{2 \alpha},
$$

and free-surface elevation

$$
h(x, t)=-t+\frac{2 \alpha x}{\pi} \arcsin \left(\frac{c(t)}{x}\right),
$$

for $|x|>c(t)$.

For wedge impact, there are again many similarities in the behavior predicted by the two models. The horizontal position of $c_{\text {gas }}(t)$ and $c(t)$ is similar throughout the impact evolution, with $E=10.89 \%$ at touchdown in the gas-cushioned model without surface tension. This gives similar horizontal locations for the maximum pressure in both theories. For wedge impact, the downwards velocity of the liquid free-surface in the trapped gas region is even closer to the descent speed of the solid body, than to the impact of a quadratic shaped body. As before, this similarity is due to the gas surrounding the tip of the wedge being unable to drain away after the pressure bifurcates forming a pair of local pressure maxima where there is no gas flux. On the liquid free-surface, the liquid velocity potentials are of comparable magnitude. At the time of touchdown in a gas-cushioned impact, the liquid velocity potential at the wedge tip is slightly more negative than the corresponding prediction of Wagner theory, while the non-zero pressures throughout the spatial domain in a gas-cushioned impact smooth the liquid velocity potential profiles so they no longer exhibit the cusp at the contact line associated with Wagner theory. At $x=0$ and touchdown, the differences in the liquid velocity potentials at the wedge tip are less than the differences in the liquid velocity potential at $t=0$.

Gas-cushioned wedge impacts with surface tension are shown in Fig. 7 with $\alpha=4$ and (a) $\sigma=0$, (b) $\sigma=0.02$, and (c) $\sigma=0.04$. In (b), capillary waves form on the liquid free-surface, which decay as $|x| \rightarrow \infty$, matching the waves seen for a quadratic body shape. Values of the surface tension parameter close to $\sigma=0.02$ act to delay touchdown beyond the time predicted in the absence of surface tension, echoing the behavior seen for a quadratic body shape. However, further increases in surface tension act to smooth the rapid changes in the free-surface height at $x=0$, which are observed for $\sigma=0$. For $\sigma=0.04$, this locally reduces the vertical separation between the wedge tip and the liquid free-surface, initiating an additional local pressure buildup in the centre of the gas cushion that drives gas away from the wedge tip. For $\sigma=0.04$, the liquid free-surface is still initially deflected downwards by a pressure buildup as the wedge moves toward touchdown. However, the pressure and the free surface do not bifurcate, with the largest pressures occurring at $x=0$ throughout the cushioning period. Initial touchdown occurs at the tip of the wedge, and a pocket of trapped gas is not formed. Touchdown is less delayed than in gas-cushioned impacts without surface tension or in gas-cushioned impacts with smaller values of $\sigma$ as surface tension reduces the liquid free-surface deflection, leading to a concomitant reduction in the time taken for the wedge to traverse the gas film.

\section{Power-law body}

The impact of a body which can be expressed by the power law $s(x)=\alpha|x|^{\beta}$ is now investigated for $\alpha>0$ and $\beta>0$. Drop tests ${ }^{39}$ and numerical simulations ${ }^{40}$ involving curved wedges, with shapes similar to this, have been conducted. However, the minimum mean deadrise angle in these experiments was $25^{\circ}$ and gas entrainment was not observed. The quadratic and wedge-shaped bodies investigated so far are special cases of this body shape, with $\beta=2$ and $\beta=1$, respectively. Substituting this body shape into Eq. (26b) gives the contact line position

$$
c(t)=\left(\frac{\sqrt{\pi}}{\alpha} \frac{\Gamma\left(\frac{2+\beta}{2}\right)}{\Gamma\left(\frac{1+\beta}{2}\right)} t\right)^{1 / \beta} .
$$

The derivation of this expression is described in Appendix A. Evaluating the gamma functions $\Gamma(1)=1, \Gamma\left(\frac{3}{2}\right)=\frac{1}{2} \sqrt{\pi}$, and $\Gamma(2)=1$ 
(a)
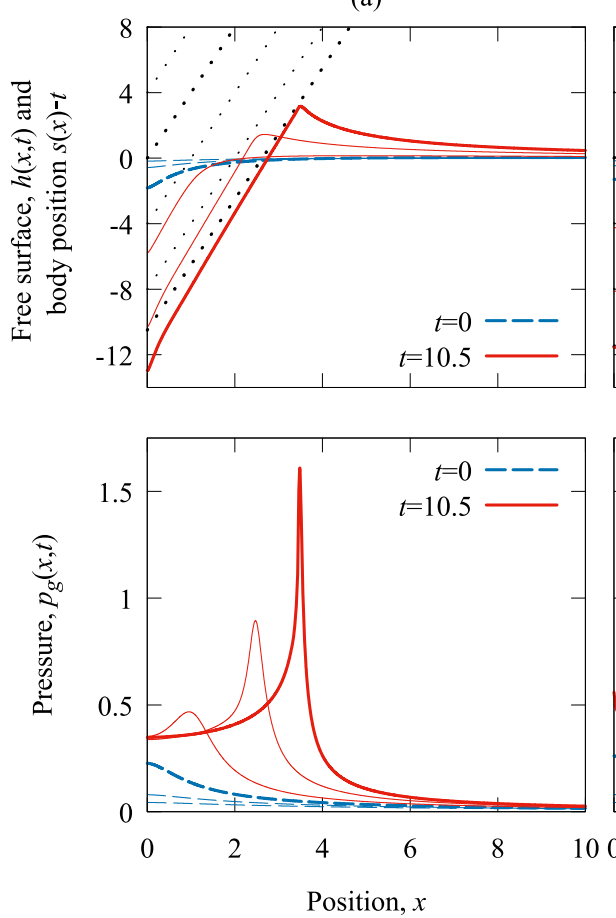

(b)
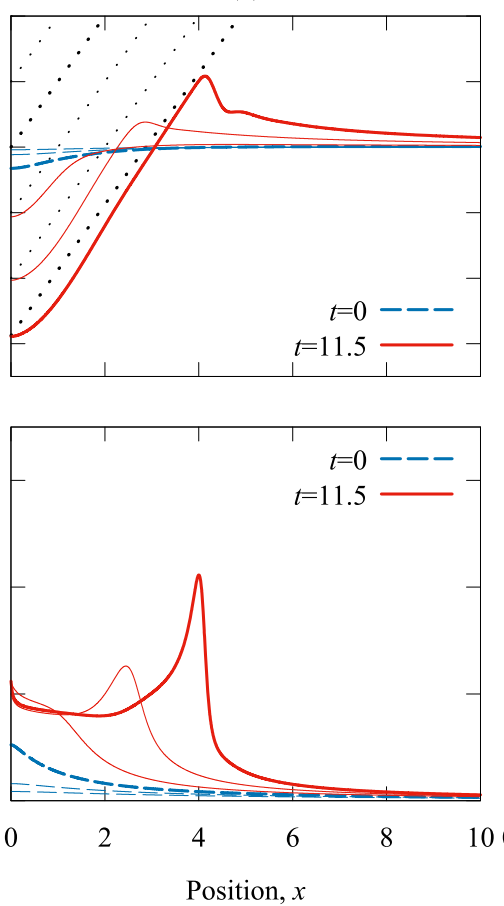

(c)
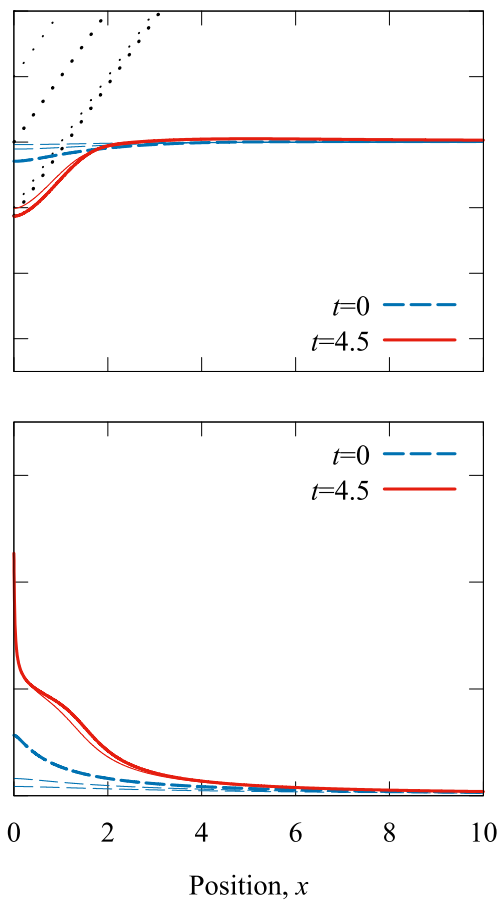

FIG. 7. Gas cushioned impact of a wedge-shaped body $s(x)=4|x|$ (black dotted line) with surface tension parameters (a) $\sigma=0$, (b) $\sigma=0.02$, and (c) $\sigma=0.04$. Profiles are shown at every fourth non-dimensional integer time step except for the final profile (thick solid red line), which is shown immediately before touchdown. In the absence of gas cushioning, touchdown occurs at $t=0$ (thick dashed blue line).

facilitates the recovery of Eq. (27a) for $\beta=2$, while Eq. (31a) is recovered when $\beta=1$. Similarly, substituting the body shape into Eq. (26a) gives the free-surface position

$$
h(x, t)=\frac{t \sqrt{x^{2}-c(t)^{2}}}{|x|}{ }_{2} F_{1}\left(1, \frac{1+\beta}{2} ; \frac{2+\beta}{2} ; \frac{c(t)^{2}}{x^{2}}\right)-t,
$$

for $|x|>c(t)$, where ${ }_{2} F_{1}(\cdot, \cdot ; \cdot ; \cdot)$ is a hypergeometric function. The derivation of this free-surface height is presented in Appendix B.

Figure 8 illustrates the solid body $s(x)=|x|^{3 / 2}$, with the freesurface height predicted by Wagner theory shown for $x<0$ and viscous gas pre-impact cushioning with $\sigma=0$ shown for $x>0$. The evolution of the free surface pressure, velocity, and liquid velocity potential is not shown, but these are consistent with previous results. In Wagner theory, an unbounded pressure [given by Eq. (19)] is predicted at the contact line, while for gas-cushioned impacts, the pressure maximum bifurcates alongside the liquid free-surface, leading to local pressure maxima at the point of minimum separation between the solid and the liquid free-surface. In the absence of surface tension, $E=6.74 \%$ at touchdown.

For the wedge $(\beta=1)$, the far-field pressure decays like $p_{g} \sim x^{-1}$ as $x \rightarrow \infty$. For $\beta<1$, the pressure decays more slowly than this, and numerical solutions of the gas cushioning model satisfying the far-field conditions could not be obtained. While numerical solutions for gas-cushioned impacts are only available for $\beta \geq 1$, the integral in Eq. (26a) exists for $\beta>-1$ (see Appendix A). However, physically realizable body shapes with a bounded global minimum require $\beta>0$. Although numerical solutions with gas cushioning could not be obtained for $0<\beta<1$, the Wagner theory prediction for the free-surface height (33) is valid in this range. As an example

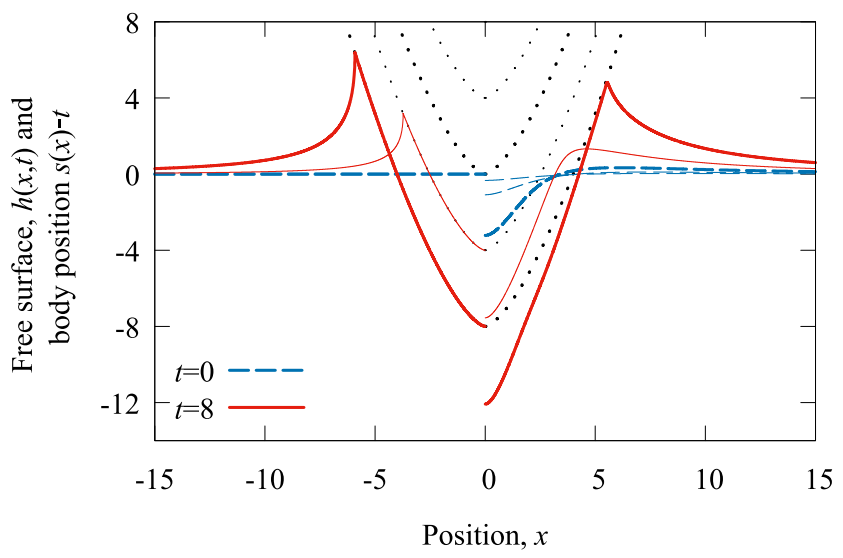

FIG. 8. Free-surface and body position for the impact of the body $s(x)=|x|^{3 / 2}$ (black dotted line) with an initially quiescent liquid. The Wagner solution is shown for $x<0$, while numerical solutions for viscous gas cushioning is shown for $x>0$. Profiles are shown at every fourth non-dimensional integer time step, with the final profile at $t=8$ (thick solid red line), when touchdown occurs with gas cushioning. In the absence of gas cushioning, touchdown occurs at $t=0$ (thick dashed blue line). 
of free-surface profiles in this range, the free-surface profiles produced by the impact of a body with shape $s(x)=|x|^{1 / 2}$ are shown in Fig. 9.

As with the quadratic-shaped body and the wedge, there is good agreement between the horizontal positions of $c(t)$ and $c_{\text {gas }}(t)$ when $\sigma=0$. This similarity is obtained for body shapes $s(x)=\alpha|x|^{\beta}$ with $\alpha=1$. However, the Wagner wetted region and the extent of the gas bubble both depend upon the body shape parameter $\alpha$. As $\alpha$ increases, the horizontal extent and area of the trapped gas pocket decreases as the horizontal extent of the body within the gas cushioning region also decreases. Numerical solutions for the gas cushioning model are only available for $\beta \geq 1$, and in this regime, the dependence of the solution profiles upon $\alpha$ can be codified by scaling lengths and time by

$$
(h, s, t, x)=\left(\alpha^{\kappa} \bar{h}, \alpha^{\kappa} \bar{s}, \alpha^{\kappa} \bar{t}, \alpha^{2 \kappa} \bar{x}\right),
$$

where $\kappa=-1 /(2 \beta-1)$, alongside rescaled pressures and a rescaled surface tension coefficient

$$
\left(p_{g}, p_{l}, \sigma\right)=\left(\alpha^{\kappa} \bar{p}_{g}, \alpha^{\kappa} \bar{p}_{l}, \alpha^{4 \kappa} \bar{\sigma}\right) .
$$

This rescaling eliminates $\alpha$ from the body shape and Eqs. (6)-(8). In the absence of surface tension, this scaling indicates that if the value of $\alpha$ is doubled, then for a wedge (with $\beta=1$ ), the horizontal extent of the trapped gas pocket decreases by a factor of four, while the area enclosed between the solid body and the liquid free-surface decreases by a factor of eight. For a quadratic parabola (with $\beta=2$ ), the horizontal extent of the trapped gas pocket decreases by a factor of $2^{2 / 3}$, while the area enclosed between the solid body and the liquid free-surface decreases by a factor of two.

The horizontal extent of the wetted region also decreases as $\alpha$ increases as the body rises out of the liquid more rapidly. The parameter $\alpha$ is removed from the body shape and is not introduced into the Wagner contact line position and the Wagner free-surface height by defining a rescaled free-surface height $\hat{h}$, a body height $\hat{s}$, and time $\hat{t}$,

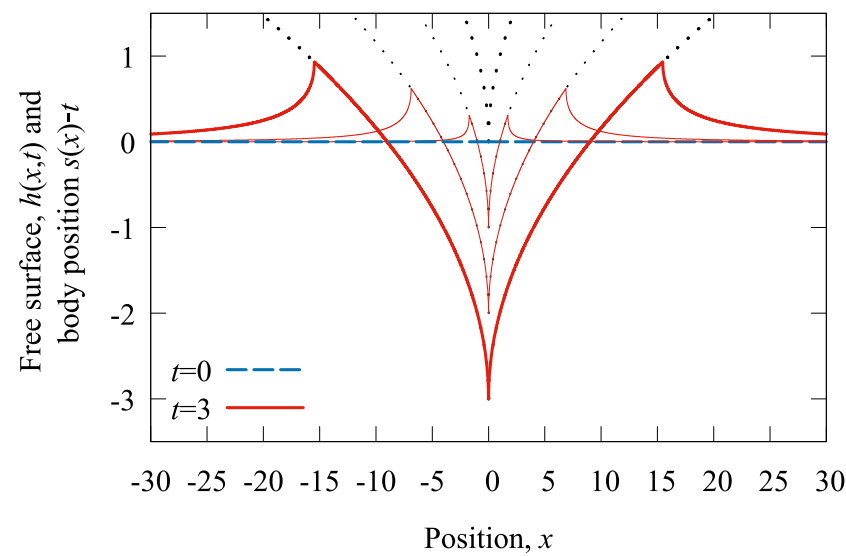

FIG. 9. The body position and Wagner solution for free-surface for the impact of the body $s(x)=|x|^{1 / 2}$ (black dotted line). Profiles are shown at every nondimensional integer time step, from $t=0$ (thick dashed blue line) until $t=3$ (thick solid red line). which satisfy

$$
(h, s, t)=(\alpha \hat{h}, \alpha \hat{s}, \alpha \hat{t}) .
$$

As this rescaled time differs from the rescaled time associated with viscous gas cushioned impacts, the results of the two theories are no longer directly comparable without further rescaling of time. However, at a common time $t=\alpha \hat{t}=\alpha^{\kappa} \bar{t}$, corresponding to the touchdown time with gas cushioning, if the Wagner contact line position from Eq. (32) is expressed in terms of the rescaled gas cushioning time $\bar{t}$, then $c(\bar{t}) \sim \alpha^{-2 /(2 \beta-1)}$. In the gas cushioning model, the position of $c_{\text {gas }}(t)$ is expected to scale like the horizontal spatial coordinate and so from Eq. (34a), $c_{\text {gas }} \sim x \sim \alpha^{-2 /(2 \beta-1)}$. Consequently, for variations in $\alpha$ and fixed $\beta>1$ (when numerical solutions with gas cushioning are obtained), the horizontal extent of the gas pocket and the horizontal extent of the Wagner wetted region remain similar at the touchdown time of the gas-cushioned impact.

\section{Flat-bottomed wedge body}

The final body shape investigated is a flat-bottomed wedge $s(x)=\max (\alpha|x|-\beta, 0)$, for $\alpha>0$ and $\beta>0$. Impact experiments with this body shape show that touchdown initially occurs at the transition between the flat bottom and the sloping side of the wedge. ${ }^{41}$ Gas cushioning models for this body shape have previously only considered inviscid gas, ${ }^{4,16}$ while these models also assume the deadrise angle of the wedge is large so that the body can be approximated by a flat plate with the gas pressures tending to zero at the edge of the flat bottom. With these assumptions, analytic expressions for small deformations of the free-surface profile have been obtained, which are in qualitative agreement with experiments. However, if the sloping sides of a flat-bottomed wedge have a large deadrise angle, then the modeling is also valid for the impact of a flat plate. Impact experiments with a flat plate indicate that initial touchdown also occurs at the plate edge, $, 42,43$ while modeling of a flat plate impact in the inviscid gas regime also predicts touchdown in this location. ${ }^{15}$ Here, we differ from earlier studies by assuming viscous gas cushioning and allowing smaller deadrise angles on the wedge. Smaller deadrise angles enable non-zero gas pressures outside the flat-bottom region.

In the absence of gas cushioning, this body shape touches down at $t=0$ throughout the region $|x|<\beta / \alpha$. For $t>0$, the position of the contact line $c(t)$ is given as the solution of the transcendental equation ${ }^{4,5}$

$$
\frac{\pi t}{2}-\sqrt{\alpha^{2} c(t)^{2}-\beta^{2}}+\beta\left(\frac{\pi}{2}-\arcsin \left(\frac{\beta}{\alpha c(t)}\right)\right)=0,
$$

which in general must be solved numerically. However, once $c(t)$ has been determined, the horizontal velocity of the contact line is given by

$$
\dot{c}(t)=\frac{\pi c(t)}{2 \sqrt{\alpha^{2} c(t)^{2}-\beta^{2}}} .
$$

For $t$ large, the proportion of the wetted surface corresponding to the flat bottom of the wedge decreases, and in this limit, the velocity of the contact line approaches the contact line velocity for a wedge [given by the derivative of Eq. (31a) with respect to $t$ ]. The 
corresponding liquid free-surface height is given by

$$
\begin{aligned}
h(x, t)= & -t-\beta+\frac{\alpha x+\beta}{\pi} \arcsin \left(\frac{\beta x+\alpha c(t)^{2}}{c(t)(\alpha x+\beta)}\right) \\
& -\frac{\alpha x-\beta}{\pi} \arcsin \left(\frac{\beta x-\alpha c(t)^{2}}{c(t)(\alpha x-\beta)}\right),
\end{aligned}
$$

for $|x|>c(t)>\beta / \alpha$.

A comparison of the free-surface position and velocity predicted by Wagner theory and the viscous gas cushioned impact model is shown in Fig. 10 for $\alpha=1, \beta=15$, and $\sigma=0$. In viscous gas cushioned impacts, again there is a buildup in the gas pressure (not shown) that deflects the liquid free-surface downwards out of the path of the impactor. This leads to a subsequent bifurcation of both the free-surface position and the pressure, with maxima in the pressure and minima in the vertical separation between the body and liquid free-surface located at some position along the inclined side of the flat-bottomed wedge.

The minima in the vertical separation between the solid body and the liquid free-surface on the inclined side of the flat-bottomed wedge are not the only minima in the vertical separation. The inset
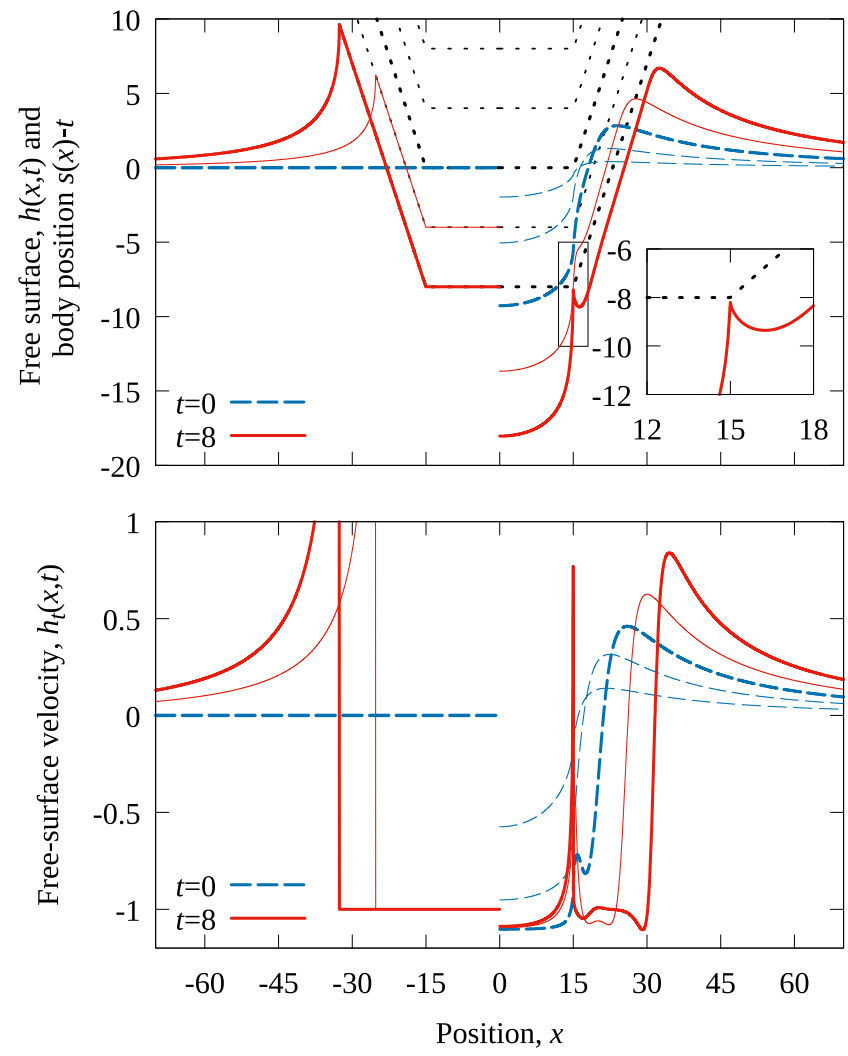

FIG. 10. Free-surface and body position (top), and free-surface velocity (bottom), for the impact of a flat-bottomed wedge with $\alpha=1$ and $\beta=15$ (black dotted line). The Wagner solution is shown for $x<0$, while the solution with viscous gas cushioning is shown for $x>0$. Profiles are shown at every fourth non-dimensional integer time step, with the final profile immediately before touchdown (thick solid red line). In the absence of gas cushioning, touchdown occurs at $t=0$ (thick dashed blue line) in Fig. 10 (top) shows the free-surface profile in the gas cushioning model, close to the corner of the solid body at $x=\beta / \alpha$. At this point, the liquid free-surface is deflected more slowly out of the path of the solid body, and hence there is a local thinning of the gas cushion, driven by the change in gradient of the solid body. There are now two possible touchdown locations: on the sloping sides of the wedge or at the corner of the body. The location of the touchdown point depends on the values of $\alpha$ and $\beta$. Touchdown at the transition between the flat bottom and sloping wedge sides is consistent with experiments using flat-bottom wedges, ${ }^{41}$ while touchdown at the edge of the plate is also observed in experiments of flat plate impact. $^{42,43}$ At touchdown, following the behavior observed with other body shapes, the horizontal location of the point of minimum gas layer thickness on the sloping side of the wedge is broadly comparable to $c(t)$ although the horizontal extent of the wetted region predicted by Wagner theory is slightly larger.

Figure 10 (bottom) shows the corresponding vertical velocity of the free surface. The maximum upwards velocity of the free surface occurs at $|x|=c(t)$ in Wagner theory [where Eq. (18) predicts an unbounded free-surface velocity], while in a viscous gas cushioned impact, the maximum upwards velocity of the liquid free-surface occurs for $|x|$ slightly larger than $c_{\text {gas }}(t)$. Once the pressure has bifurcated in the cushioning model, the pressure maxima at $x= \pm c_{\text {gas }}(t)$ again trap the gas beneath the solid body, causing the gas to descend into the liquid with the speed of the body. The predicted free-surface velocities in the trapped gas pocket match the body descent speed, except for local regions at the corner of the body $(x= \pm \beta / \alpha)$ and where the vertical velocity of the free surface rapidly transitions to positive values outside the trapped gas pocket at $x= \pm c_{\text {gas }}(t)$. The reduced free-surface descent speed at $x= \pm \beta / \alpha$ produces the local thinning of the gas cushion and touchdown observed in the free-surface height profiles.

\section{DISCUSSION AND CONCLUSIONS}

Wagner theory and a model of pre-impact viscous gas cushioning have been compared for the normal impact of a symmetric body with an initially quiescent liquid. Particular attention has been paid to the time period between $t=0$ (when touchdown occurs in Wagner theory) and the delayed initial touchdown with gas cushioning when both theories are valid. A range of solid body shapes have been investigated, including wedges and flat-bottom wedges (where viscous gas cushioning has not been previously investigated), and a generalized power law body shape $s(x)=\alpha|x|^{\beta}$ (for which both gas cushioning and the Wagner outer solution are novel).

Across the range of body shapes investigated, commonalities between the free-surface and pressure profiles generated by gas cushioning and Wagner theory are found. Driving these commonalities is the distribution of the pressure buildup induced either by preimpact cushioning or, in Wagner theory, by the liquid-solid impact itself. These commonalities are listed as follows:

- The horizontal location of touchdown with gas cushioning is broadly similar to the contact line position in Wagner theory at the corresponding time. For the body $s(x)=\alpha|x|^{\beta}$, scaling laws show that both $c(t)$ and $c_{\text {gas }}(t)$ have the same dependence on $\alpha$, so the similarity between $c(t)$ and $c_{\text {gas }}(t)$ at touchdown is preserved as $\alpha$ varies. 
- The gas cushioning pressure (which is largest where the separation between impactor and liquid is least) decays gradually to the far-field pressure, spreading the pressure buildup beyond the Wagner wetted region. Gas cushioning pressures remain bounded throughout the cushioning phase of the impact and regularize the unbounded pressure profiles predicted by the Wagner outer problem.

- Just prior to touchdown in the gas cushioning model, the highest pressures are obtained away from the global body minimum, at $c(t)$ in Wagner theory and at $c_{\text {gas }}(t)$ with preimpact gas cushioning. This is because a large pressure gradient is required at these points to induce motion in the liquid to move it out of the path of the impactor.

- Pressure profiles obtained from Wagner theory and preimpact cushioning, which have comparable support, common magnitude $\left(\rho_{l} V^{2} / \varepsilon\right)$, and maxima at the same horizontal locations, will generate similar responses in the liquid free-surface and vertical velocities.

Surface tension in pre-impact viscous gas cushioning is also investigated and is shown to smooth rapid variations in the freesurface profile. With the inclusion of surface tension:

- Capillary waves are formed across a range of body shapes. For smooth body shapes, the delay in impact due to gas cushioning is increased as the cusp on the free surface associated with touchdown is unable to form. ${ }^{3}$

- The deflection of the free-surface for body shapes with a sharp corner is reduced. This can facilitate more efficient gas film drainage as rapid changes in the gradient of the body induce additional local pressure increases. Consequently, for a wedge, surface tension can hasten touchdown relative to the time predicted in the absence of surface tension.

Having compared models of viscous gas cushioned pre-impact and Wagner theory, we can now describe the conditions where the entrainment of a gas bubble is expected to significantly affect the impact. From Eq. (28a), the horizontal extent of the trapped bubble grows as $L$ increases (as more of the body is in the cushioning region) and shrinks as $V$ increases (as less time is available before impact for the free surface to deform). If the primary interest is the pressure on the body at horizontal distances much greater than $r_{p}$ from the body minimum, then models of pre-impact gas cushioning become less useful as predictive tools as the maximum pressures at the point of interest will only be experienced some time after touchdown when the pre-impact cushioning model ceases to be valid. In this situation, Wagner theory, or some alternative theory that includes post-impact gas cushioning, is required.

Post impact behavior with gas cushioning has previously been investigated with both viscous ${ }^{18}$ and inviscid ${ }^{27,28}$ gas. However, these models are initiated with the global body minimum in contact with the liquid. As the body does not move toward the liquid in the pre-impact phase, there is no opportunity for the pressure to build up prior to touchdown. Consequently, the liquid is at rest, with an undisturbed free surface until touchdown, even though the models include the influence of air. While there are scenarios where an initially stationary body, in contact with a liquid, starts to move into the liquid, this configuration is not common for liquid-solid impacts as the body is usually in motion before touchdown. Further work is required to couple pre- and post-impact gas effects in a model that allows body motion in the pre-impact phase. Such a model would extend the time period over which gas cushioning can be investigated, enabling a comparison of the effects of gas cushioning and Wagner theory throughout the initial stages of the impact and not just until the time the body first touches down.

\section{ACKNOWLEDGMENTS}

Snizhana Ross was supported by the Development Trust of the University of Aberdeen.

\section{APPENDIX A: DERIVATION OF THE CONTACT-LINE POSITION FOR A POWER-LAW BODY}

For the body shape $s(x)=\alpha|x|^{\beta}$, the consistency condition (26b) implies

$$
\alpha c(t)^{\beta} \int_{0}^{1} \frac{\Xi^{\beta} \mathrm{d} \Xi}{\sqrt{1-\Xi^{2}}}=\frac{\pi t}{2} .
$$

From equation 8.380 .1 by Gradshteyn and Ryzhik, ${ }^{44}$ the beta function $B(p, q)$ satisfies

$$
B(p, q)=2 \int_{0}^{1} \Xi^{2 p-1}\left(1-\Xi^{2}\right)^{y-1} \mathrm{~d} \Xi,
$$

for $\operatorname{Re}[p]>0$ and $\operatorname{Re}[q]>0$. If we choose $p=(1+\beta) / 2$ and $q=1 / 2$, then Eq. (A1) implies

$$
\alpha c(t)^{\beta} B\left(\frac{1+\beta}{2}, \frac{1}{2}\right)=\pi t
$$

for $\beta>-1$. In this expression, the beta function can be written in terms of gamma functions as $B\left(\frac{1+\beta}{2}, \frac{1}{2}\right)=\Gamma\left(\frac{1+\beta}{2}\right) \Gamma\left(\frac{1}{2}\right) / \Gamma\left(\frac{2+\beta}{2}\right)$. Equation (32) is recovered by noting that $\Gamma\left(\frac{1}{2}\right)=\sqrt{\pi}$.

\section{APPENDIX B: DERIVATION OF THE FREE-SURFACE HEIGHT FOR A POWER-LAW BODY}

For the body shape $s(x)=\alpha|x|^{\beta}$, the free-surface position (26a) can be expressed in terms of the hypergeometric function ${ }_{2} F_{1}(a, b ; c ; z)$, as given in Eq. (33). An integral representation of the hypergeometric function is given by Abramowitz and Stegun equation 15.3.1, ${ }^{45}$ with the form

$$
{ }_{2} F_{1}(a, b ; c ; z)=\frac{\Gamma(c)}{\Gamma(b) \Gamma(c-b)} \int_{0}^{1} t^{b-1}(1-t)^{c-b-1}(1-t z)^{-a} \mathrm{~d} t
$$

where $\operatorname{Re}[c]>\operatorname{Re}[b]>0$ and $|\arg (1-z)|<\pi$. The free surface occupying the range $x>c(t)$ (i.e., $X>1$ ) is considered first. With the substitutions $t=\Xi^{2}, z=1 / X^{2}, a=1, b=(1+\beta) / 2$, and $c=(2$ $+\beta) / 2$, this integral becomes

$$
\int_{0}^{1} \frac{\Xi^{\beta} \mathrm{d} \Xi}{\sqrt{1-\Xi^{2}}\left(\Xi^{2}-X^{2}\right)}=-\frac{\sqrt{\pi} \Gamma\left(\frac{1+\beta}{2}\right)}{2 X^{2} \Gamma\left(\frac{2+\beta}{2}\right)}{ }_{2} F_{1}\left(1, \frac{1+\beta}{2} ; \frac{2+\beta}{2} ; \frac{1}{X^{2}}\right) .
$$


As $X>1$, the conditions on Eq. (B1) are satisfied for $\beta>-1$, which covers all cases of interest. Upon substituting this integral into Eq. (26a) and using the contact line position (32), an expression for the free-surface profile for $x>c(t)$ is recovered. Similar considerations for $x<-c(t)$ (i.e., $X<-1$ ) give rise to Eq. (33).

Using Mathematica, ${ }^{46}$ the hypergeometric function in Eq. (33) can be expressed in terms of common functions for some specific values of $\beta$. For a quadratic body, $\beta=2$ and

$$
{ }_{2} F_{1}\left(1, \frac{3}{2} ; 2 ; \frac{c(t)^{2}}{x^{2}}\right)=-\frac{2 x^{2}\left(\sqrt{x^{2}-c(t)^{2}}-|x|\right)}{c(t)^{2} \sqrt{x^{2}-c(t)^{2}}},
$$

while for a wedge body, $\beta=1$ and

$$
{ }_{2} F_{1}\left(1,1 ; \frac{3}{2} ; \frac{c(t)^{2}}{x^{2}}\right)=\frac{x|x| \arcsin \left(\frac{c(t)}{x}\right)}{c(t) \sqrt{x^{2}-c(t)^{2}}}
$$

enabling the recovery of Eqs. (27b) and (31b), respectively.

\section{REFERENCES}

${ }^{1} \mathrm{H}$. Wagner, "Über stoß- und gleitvorgänge an der oberfläche von flüssigkeiten (Phenomena associated with impacts and sliding on liquid surfaces)," Z. Angew. Math. Mech. 12, 193-215 (1932).

${ }^{2}$ R. Cointe and J.-L. Armand, "Hydrodynamic impact analysis of a cylinder," J. Offshore Mech. Arct. Eng. 109, 237-243 (1987).

${ }^{3}$ A. A. Korobkin and V. V. Pukhnachov, "Initial stage of water impact," Annu. Rev. Fluid Mech. 20, 159-185 (1988).

${ }^{4}$ S. D. Howison, J. R. Ockendon, and S. K. Wilson, "Incompressible waterentry problems at small deadrise angles," J. Fluid Mech. 222, 215-230 (1991).

${ }^{5}$ J. M. Oliver, "Water entry and related problems," Ph.D. thesis, University of Oxford, Oxford, United Kingdom, 2002.

${ }^{6}$ A. A. Korobkin and A. Iafrati, "Hydrodynamic loads during initial stage of floating body impact," J. Fluids Struct. 21, 413-427 (2005).

${ }^{7}$ R. Purvis and F. T. Smith, "Droplet impact on water layers: Post-impact analysis and computations," Philos. Trans. R. Soc., A 363, 1209-1221 (2005).

${ }^{8}$ S. D. Howison, J. R. Ockendon, J. M. Oliver, R. Purvis, and F. T. Smith, "Droplet impact on a thin fluid layer," J. Fluid Mech. 542, 1-23 (2005).

${ }^{9}$ J. Philippi, P.-Y. Lagrée, and A. Antkowiak, "Drop impact on a solid surface: Short-time self-similarity,” J. Fluid Mech. 795, 96-135 (2016).

${ }^{10}$ S.-L. Chuang, "Slamming of rigid wedge-shaped bodies with various deadrise angles," Technical Report 2268, Department of the Navy, Structural Mechanics Laboratory, USA, 1966.

${ }^{11} \mathrm{~K}$. Hagiwara and T. Yuhara, "Fundamental study of wave impact load on ship bow (1st report): Maximum impact pressures acting on a semi-cylindrical body like bow of a large ship," J. Soc. Nav. Archit. Jpn. 1974, 181-189.

${ }^{12}$ F. J. Huera-Huarte, D. Jeon, and M. Gharib, "Experimental investigation of water slamming loads on panels," Ocean Eng. 38, 1347-1355 (2011).

${ }^{13}$ D. H. Peregrine, "Water wave impact on walls," Annu. Rev. Fluid Mech. 35, 23-43 (2003).

${ }^{14}$ O. M. Faltinsen and A. N. Timokha, Sloshing (Cambridge University Press, Cambridge, 2009).

${ }^{15}$ J. H. G. Verhagen, "The impact of a flat plate on a water surface," J. Ship Res. 11, 211-223 (1967).

${ }^{16} \mathrm{~S}$. K. Wilson, "A mathematical model for the initial stages of fluid impact in the presence of a cushioning fluid layer," J. Eng. Math. 25, 265-285 (1991).

${ }^{17}$ F. T. Smith, L. Li, and G. X. Wu, "Air cushioning with a lubrication/inviscid balance," J. Fluid Mech. 482, 291-318 (2003).

${ }^{18}$ R. Purvis and F. T. Smith, “Air-water interactions near droplet impact," Eur. J. Appl. Math. 15, 853-871 (2004).
${ }^{19}$ P. D. Hicks and R. Purvis, "Air cushioning and bubble entrapment in threedimensional droplet impacts," J. Fluid Mech. 649, 135-163 (2010).

${ }^{20}$ P. D. Hicks and R. Purvis, "Liquid-solid impacts with compressible gas cushioning," J. Fluid Mech. 735, 120-149 (2013).

${ }^{21}$ S. Mandre, M. Mani, and M. P. Brenner, "Precursors to splashing of liquid droplets on a solid surface," Phys. Rev. Lett. 102, 134502 (2009).

${ }^{22}$ P. D. Hicks and R. Purvis, "Gas-cushioned droplet impacts with a thin layer of porous media," J. Eng. Math. 102, 65-87 (2017).

${ }^{23}$ P. D. Hicks, E. V. Ermanyuk, N. V. Gavrilov, and R. Purvis, "Air trapping at impact of a rigid sphere onto a liquid," J. Fluid Mech. 695, 310-320 (2012).

${ }^{24}$ W. Bouwhuis, M. H. W. Hendrix, D. van der Meer, and J. H. Snoeijer, "Initial surface deformations during impact on a liquid pool," J. Fluid Mech. 771, 503-519 (2015).

${ }^{25}$ L. Xu, W. W. Zhang, and S. R. Nagel, "Drop splashing on a dry smooth surface," Phys. Rev. Lett. 94, 184505 (2005).

${ }^{26}$ E. Q. Li, K. R. Langley, Y. S. Tian, P. D. Hicks, and S. T. Thoroddsen, "Double contact during drop impact on a solid under reduced air pressure," Phys. Rev. Lett. 119, 214502 (2017).

${ }^{27}$ M. R. Moore, J. R. Ockendon, and J. M. Oliver, "Air-cushioning in impact problems," IMA J. Appl. Math. 78, 818-838 (2013).

${ }^{28}$ M. R. Moore and J. M. Oliver, "On air cushioning in axisymmetric impacts," IMA J. Appl. Math. 79, 661-680 (2014).

${ }^{29}$ A. A. Korobkin, "Inclined entry of a blunt profile into an ideal fluid," Fluid Dyn. 23, 443-447 (1988).

${ }^{30}$ A. A. Korobkin, "The entry of an elliptical paraboloid into a liquid at variable velocity," J. Appl. Math. Mech. 66, 39-48 (2002).

${ }^{31}$ F. D. Gakhov, Boundary Value Problems (Dover, Mineola, New York, 1966).

${ }^{32}$ L. Duchemin and C. Josserand, "Curvature singularity and film-skating during drop impact," Phys. Fluids 23, 091701 (2011).

${ }^{33}$ J. O. Marston, I. U. Vakarelski, and S. T. Thoroddsen, "Bubble entrapment during sphere impact onto quiescent liquid surfaces," J. Fluid Mech. 680, 660-670 (2011).

${ }^{34} \mathrm{E}$. Q. Li and S. T. Thoroddsen, "Time-resolved imaging of a compressible air disc under a drop impacting on a solid surface," J. Fluid Mech. 780, 636-648 (2015).

${ }^{35} \mathrm{~S}$. Okada and Y. Sumi, "On the water impact and elastic response of a flat plate at small impact angles," J. Mar. Sci. Technol. 5, 31-39 (2000).

${ }^{36} \mathrm{M}$. Greenhow, "Wedge entry into initially calm water," Appl. Ocean Res. 9, 214-223 (1987)

${ }^{37}$ T. Tveitnes, A. C. Fairlie-Clarke, and K. Varyani, "An experimental investigation into the constant velocity water entry of wedge-shaped sections," Ocean Eng. 35, 1463-1478 (2008).

${ }^{38}$ M. Barjasteh, H. Zeraatgar, and M. J. Javaherian, "An experimental study on water entry of asymmetric wedges," Appl. Ocean Res. 58, 292-304 (2016).

${ }^{39}$ R. Panciroli, A. Shams, and M. Porfiri, "Experiments on the water entry of curved wedges: High speed imaging and particle image velocimetry," Ocean Eng. 94, 213-222 (2015).

${ }^{40} \mathrm{P}$. Yu, H. Li, and M. C. Ong, "Numerical study on the water entry of curved wedges," Ships Offshore Struct. 13, 885-898 (2018).

${ }^{41}$ A. Driscoll and A. Lloyd, "Slamming experiments-Description of facilities and details of impact pressure results," Technical Report R82002, Admiralty Marine Technology Establishment, AMTE (Haslar), Haslar, Gosport, Hants, UK, 1982.

${ }^{42}$ S. H. Oh, S. H. Kwon, and J. Y. Chung, "A close look at air pocket evolution in flat impact," in Proceedings of the 24th International Workshop on Water Waves and Floating Bodies, 2009.

${ }^{43}$ H. C. Mayer and R. Krechetnikov, "Flat plate impact on water," J. Fluid Mech. 850, 1066-1116 (2018).

${ }^{44}$ I. S. Gradshteyn and I. M. Ryzhik, in Table of Integrals, Series and Products, edited by A. Jeffrey (Academic Press, Elsevier, Amsterdam, 2000).

${ }^{45}$ Handbook of Mathematical Functions, 10th ed., edited by M. Abramowitz and I. A. Stegun (Dover, New York, 1972).

${ }^{46}$ Wolfram Research, Mathematica, Version 11.3, Champaign, IL, USA, 2018. 